\title{
Enantioselective NHC-Catalysed Redox [4+2]-Hetero-Diels-Alder Reactions using $\alpha$-Aroyloxyaldehydes and Unsaturated Ketoesters
}

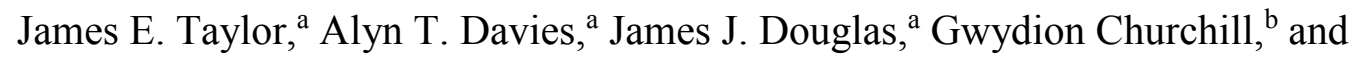
Andrew D. Smith ${ }^{\mathrm{a}, *}$

${ }^{a}$ EaStCHEM, School of Chemistry, University of St Andrews, North Haugh, St Andrews, KY16 9ST, U.K.

${ }^{\mathrm{b}}$ AstraZeneca, Process Research and Development, Macclesfield, Cheshire, SK10 2NA, U.K.

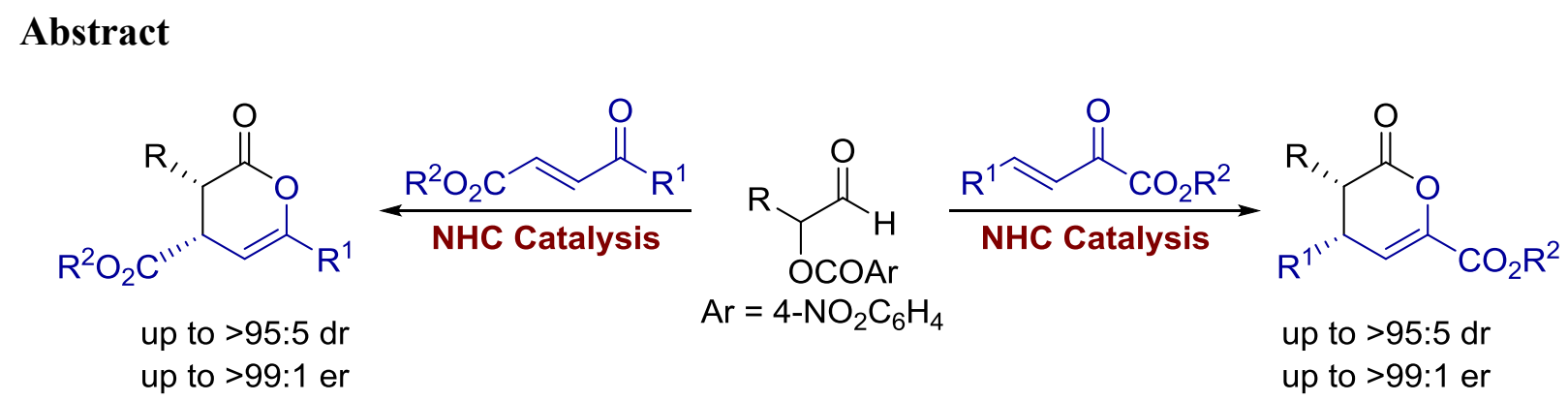

$\mathrm{N}$-Heterocyclic carbene (NHC)-catalysed redox [4+2]-hetero-Diels-Alder reactions of $\alpha$ aroyloxyaldehydes with either $\beta, \gamma$-unsaturated $\alpha$-ketoesters or $\alpha, \beta$-unsaturated $\gamma$-ketoesters generates substituted syn-dihydropyranones in good yield with excellent enantioselectivity (up to $>99: 1 \mathrm{er}$. The product diastereoselectivity is markedly dependent upon the nature of the unsaturated enone substituent. The presence of either electron-neutral or electron-rich aryl substituents gives excellent diastereoselectivity (up to $>99: 5 \mathrm{dr}$ ), while electron-deficient aryl substituents give reduced diastereoselectivity. In these cases, the syn-dihydropyranone products are more susceptible to base-promoted epimerisation at the $\mathrm{C}(4)$-position under the reaction conditions, accounting for the lower diastereoselectivity obtained. 


\section{Introduction}

$\mathrm{N}-\mathrm{Heterocyclic}$ carbenes (NHCs) are versatile organocatalysts that exhibit a wide range of reactivity. ${ }^{1}$ Of the multiple activation modes accessible through NHC catalysis, the generation and use of azolium enolates has emerged as a powerful strategy for the enantioselective synthesis of complex small-molecules. A number of methods has been developed for the formation of azolium enolate intermediates (Scheme 1a). For example, addition of an NHC into a substituted ketene directly generates an azolium enolate, ${ }^{2}$ while deprotonation of acyl azolium intermediates, themselves generated from either the reaction of NHCs with aldehydes under oxidative conditions ${ }^{3}$ or the addition of NHCs into activated carboxylic esters, ${ }^{4}$ also leads to enolate formation. Another common strategy for azolium enolate formation is the addition of NHCs into aldehydes bearing $\alpha$-reducible functional groups, which subsequently undergo a redox process to form the desired enolate. ${ }^{5-10}$ Examples of such $\alpha$-reducible aldehydes include $\alpha$-chloroaldehydes, ${ }^{6}$ enals, ${ }^{7} \alpha$-aryloxyaldehydes,${ }^{8}$ and formyl cyclopropanes ${ }^{9}$ (Scheme 1b). We have previously reported that bench-stable $\alpha$-aroyloxyaldehydes $\mathbf{1}$ are also efficient redox azolium enolate precursors. ${ }^{10}$

\section{a) Strategies towards azolium enolates}

(i)<smiles></smiles><smiles></smiles>

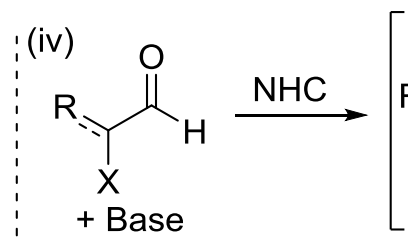

b) $\alpha$-Reducible aldehydes used as azolium enolate precursors<smiles>[R]C(Cl)C=O</smiles><smiles>[R]C=CC=O</smiles><smiles>O=CCO[N+](=O)c1ccccc1</smiles><smiles>[R]C(=O)C1CC1C=O</smiles>

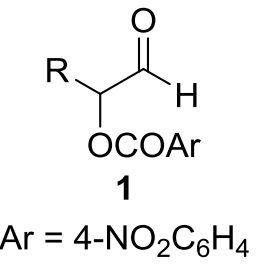

Scheme 1. Generation of azolium enolate intermediates.

Azolium enolates generated from NHCs and $\alpha$-reducible aldehydes react with a variety of electrophiles to generate a diverse array of products. Of particular relevance is the reaction of azolium enolates with substituted enones in enantioselective [4+2]-hetero-Diels-Alder processes to form stereodefined dihydropyranone products. For example, Bode and co-workers 
demonstrated that azolium enolates generated from $\alpha$-chloroaldehydes react with both $\beta, \gamma$ unsaturated $\alpha$-ketoesters and $\alpha, \beta$-unsaturated $\gamma$-ketoesters to form syn-dihydropyranones 3 with excellent enantioselectivity (Scheme $2 \mathrm{a}$ ). ${ }^{6 \mathrm{~b}}$ In this case, the use of unsaturated ketoesters bearing either alkyl or electron-neutral/rich aryl substituents generally formed the product dihydropyranones in high diastereoselectivity. Notably, the presence of electron-deficient aryl (4- $\left.\mathrm{BrC}_{6} \mathrm{H}_{4}\right)$ or heteroaryl (2-furyl) substituents lead to a decrease in diastereoselectivity, although the origin of the reduced $\mathrm{dr}$ was not further investigated. This methodology was subsequently extended to the use of the bench-stable sodium bisulfate adducts of $\alpha$ chloroaldehydes under biphasic conditions, allowing the use of unsubstituted chloroacetaldehyde sodium bisulfite to form dihydropyranones containing a single stereocentre. ${ }^{6 c}$ The NHC-catalysed redox hetero-Diels-Alder reaction of enals with unsaturated ketoesters has also been reported to proceed with high levels of stereoselectivity. ${ }^{7 \mathrm{~d}}$

$\alpha$-Aroyloxyaldehydes have previously been used as azolium enolate precursors in NHCcatalysed [4+2]-hetero-Diels-Alder reactions with various trifluoromethyl enones (Scheme $2 b)^{10 b, d}$ and $\alpha, \beta$-unsaturated trichloromethyl ketones $^{10 \mathrm{e}}$ to form functionalised 
a) Bode, 2006: NHC-catalysed [4+2] cycloaddition using a-chloroaldehydes

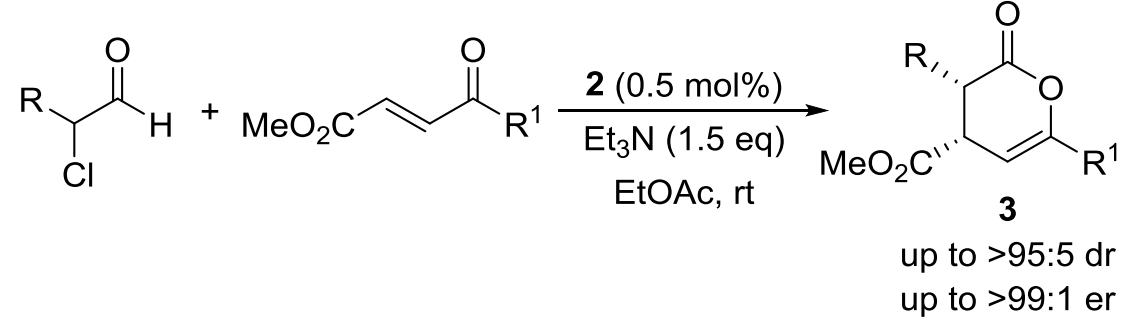

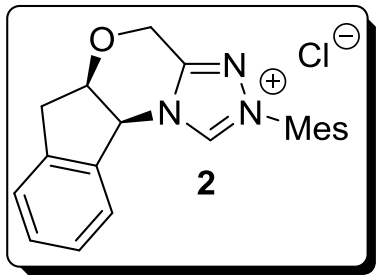

b) Smith, 2013: NHC-catalysed reaction of $\alpha$-aroyloxyaldehydes with trifluoromethyl enones<smiles>[R]C1=C[C@H](C(F)(F)F)[C@H]([R])C(=O)O1</smiles>

c) This work: NHC-catalysed reaction of $\alpha$-aroyloxyaldehydes with unsaturated ketoesters

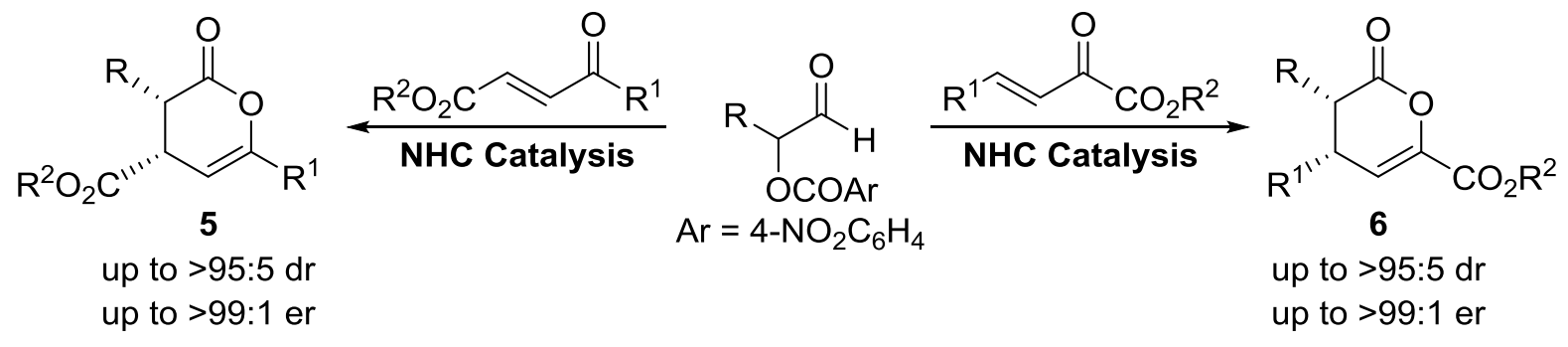

Scheme 2. NHC-catalysed redox [4+2]-hetero-Diels-Alder reactions.

syn-dihydropyranones 4 with high diastereo- and enantioselectivity. Azolium enolates generated from $\alpha$-aroyloxyaldehydes also undergo $[2+2]^{10 \mathrm{f}}$ and $[3+2]$-cycloadditions, ${ }^{10 \mathrm{~g}}$ as well as enantioselective $\alpha$-aminations to from $N$-aryl amino acid derivatives. ${ }^{10 \mathrm{c}}$

Herein, the NHC-catalysed redox hetero-Diels-Alder reaction of bench-stable $\alpha$ aroyloxyaldehydes with both $\beta, \gamma$-unsaturated $\alpha$-ketoesters and $\alpha, \beta$-unsaturated $\gamma$-ketoesters is reported to form syn-dihydropyranones (5 and 6) with excellent enantioselectivity (Scheme $2 c)$. The effect of various aryl substituents on the unsaturated ketoesters on the reaction diastereoselectivity is assessed, with control experiments performed to determine the origin of the reduced product diastereoselectivity observed in some cases.

\section{Results and discussion}

\subsection{Reaction optimisation}

First, the reaction of azolium enolates derived from $\alpha$-aroyloxyaldehydes ${ }^{11}$ with $\beta, \gamma$ unsaturated $\alpha$-ketoesters was studied. Treating $\alpha$-aroyloxyaldehyde 7 and $\alpha$-ketoester 8 with 
NHC precatalyst $2(20 \mathrm{~mol} \%)$ and $\mathrm{Et}_{3} \mathrm{~N}(1.5 \mathrm{eq})$ in $\mathrm{THF}$ at room temperature gave dihydropyranone 9 in a promising 50\% yield and 92:8 dr with excellent enantioselectivity (>99:1 er) for the major syn-diastereoisomer (Table 1, entry 1). ${ }^{12}$ Lowering the catalyst loading led to increased reaction times and decreased diastereoselectivity, although the enantioselectivity for both diastereoisomers was high (Table 1, entries 2 and 3). Decreasing the reaction temperature to $-78{ }^{\circ} \mathrm{C}$ led to an increase in diastereoselectivity ( $>99: 1 \mathrm{dr}$ ), but gave lower conversion into the desired product (Table 1, entry 4). Starting the reaction at $0{ }^{\circ} \mathrm{C}$ and allowing it to warm slowly to room temperature using $10 \mathrm{~mol} \% 2$ gave an excellent compromise between reactivity and stereoselectivity, with syn-dihydropyranone 9 isolated in $93 \%$ yield with $>95: 5 \mathrm{dr}$ and $>99: 1$ er (Table 1, entry 5 ). The relative and absolute configuration of the major product was assigned by analogy to that of a known derivative formed during investigation of the reaction substrate scope (vide infra).

\subsection{Reaction scope with $\beta, \gamma$-unsaturated $\alpha$-ketoesters}

The scope of the NHC-catalysed redox reaction of various $\alpha$-aroyloxyaldehydes with a range of $\beta, \gamma$-unsaturated $\alpha$-ketoesters was studied under the previously optimised conditions (Table 2 ). The reaction worked well with different alkyl $\alpha$-aroyloxyaldehyde substituents, forming syn-dihydropyranones $\mathbf{1 0}$ and $\mathbf{1 1}$ in good yields with excellent levels of stereoselectivity (up to $>95: 5 \mathrm{dr}$ and $>99: 1 \mathrm{er}$ ). The reaction with $\beta, \gamma$-unsaturated $\alpha$-ketoesters bearing electron-rich

Table 1

Reaction optimisation

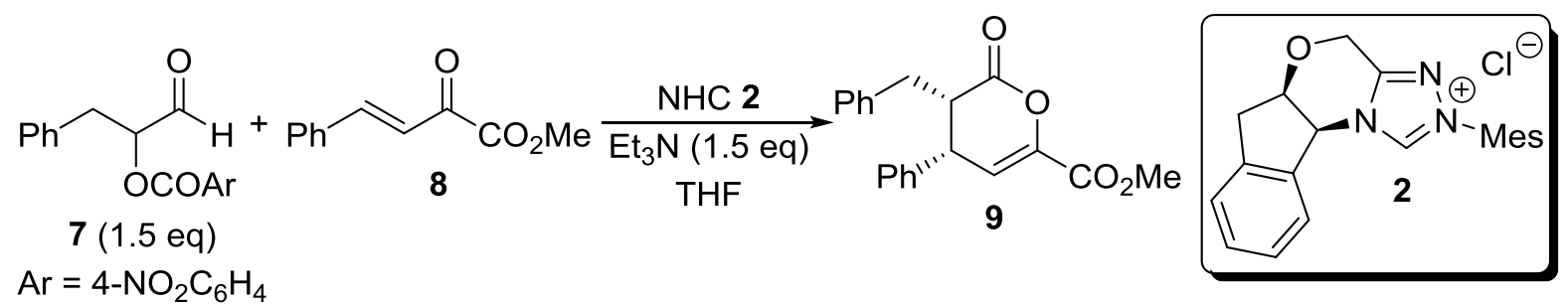

\begin{tabular}{ccccccc}
\hline Entry & NHC $(\mathrm{mol} \%)$ & $\mathrm{T}\left({ }^{\circ} \mathrm{C}\right)$ & Time $(\mathrm{h})$ & Conversion $(\%)^{\mathrm{a}, \mathrm{b}}$ & $\mathrm{dr}^{\mathrm{a}}$ & $\mathrm{er}^{\mathrm{c}}$ \\
\hline 1 & $\mathbf{2}(20)$ & $\mathrm{rt}$ & 5 & $>90(50)$ & $92: 8$ & $>99: 1$ \\
2 & $\mathbf{2}(10)$ & $\mathrm{rt}$ & 12 & $>90$ & $85: 15$ & $\mathrm{~N} / \mathrm{D}$ \\
3 & $\mathbf{2 ( 1 )}$ & $\mathrm{rt}$ & 48 & $>90$ & $76: 24$ & $>99: 1($ syn $)$ \\
4 & $\mathbf{2 ( 1 0 )}$ & -78 & 12 & 45 & $>95: 5$ & $\mathrm{~N} / \mathrm{D}$
\end{tabular}




\footnotetext{
${ }^{a}$ Determined by ${ }^{1} \mathrm{H}$ NMR analysis of the crude reaction mixture.

${ }^{\mathrm{b}}$ Isolated yield after purification by column chromatography in parentheses.

${ }^{c}$ er of major diastereoisomer determined by HPLC analysis.
}

aromatic substituents $\left(4-\mathrm{MeC}_{6} \mathrm{H}_{4}\right.$ and $\left.4-\mathrm{MeOC}_{6} \mathrm{H}_{4}\right)$ gave dihydropyranones 12-15 in high yields, but with slightly reduced levels of $s y n$-diastereoselectivity ( $\geq 91: 9 \mathrm{dr}$ ) compared with the reaction with phenyl substituted $\alpha$-ketoester $\mathbf{8}$. However, in each case the major syndiastereoisomer was formed with high levels of enantioselectivity ( $\geq 96: 4$ er). The relative configuration of syn-dihydropyranone $\mathbf{1 2}$ could be confirmed through comparison of its spectroscopic data with the literature, ${ }^{6 \mathrm{~b}}$ while the absolute configuration was confirmed through comparison of its specific rotation $\left[96: 4 \mathrm{er},[\alpha]_{\mathrm{D}}^{20}+299.4\left(c\right.\right.$ 0.5, $\left.\left.\mathrm{CHCl}_{3}\right)\right]$ with the reported value $\left[(3 S, 4 S)-\mathbf{1 2}, 98.5: 1.5 \mathrm{er},[\alpha]_{\mathrm{D}}^{20}+310.3\left(c 1.0, \mathrm{CHCl}_{3}\right)\right] .{ }^{6 b}$ The incorporation of a more electron-deficient aryl bromine substituent led to a modest $42 \%$ yield of dihydropyranone $\mathbf{1 6}$ in a reduced 89:11 dr, although the enantioselectivity remained high (99:1 er). An extended aromatic 2-naphthyl substituent was also successfully incorporated, forming 17 in $48 \%$ yield with good stereoselectivity (94:6 dr, >99:1 er). 
Table 2

Scope of the reaction using $\beta, \gamma$-unsaturated $\alpha$-ketoesters ${ }^{\mathrm{a}-\mathrm{c}}$

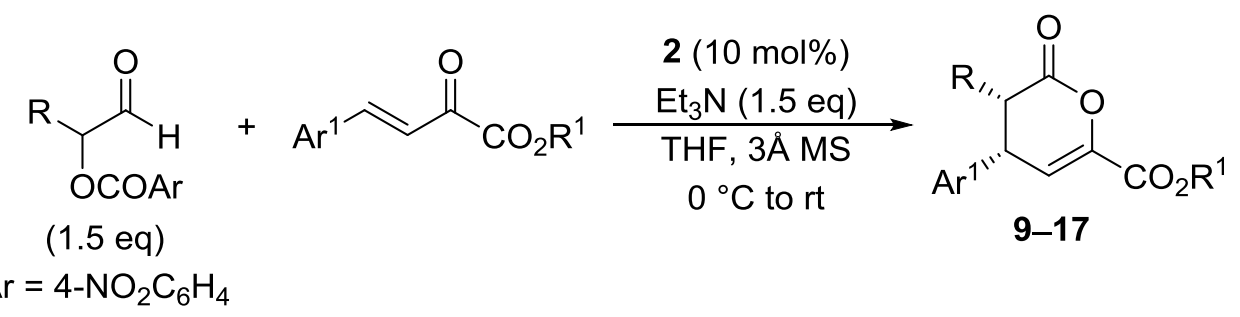<smiles>CC(=O)C1=C[C@H](c2ccccc2)[C@@H](Cc2ccccc2)C(=O)O1</smiles>

$12 \mathrm{~h}, 93 \%$ $>95: 5 \mathrm{dr},>99: 1 \mathrm{er}$<smiles>CCOC(=O)C1=C[C@@H](c2ccc(C)cc2)[C@H](Cc2ccccc2)C(=O)O1</smiles>

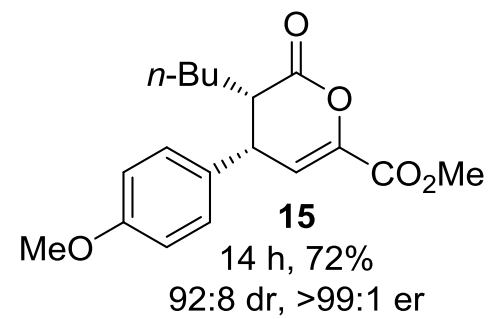

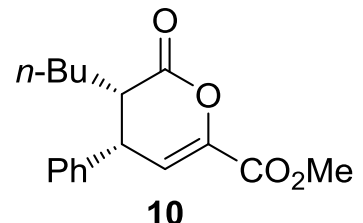

$3 \mathrm{~h}, 69 \%$ $>95: 5 \mathrm{dr}, 98: 2$ er<smiles>COC(=O)C1=C[C@H](c2ccccc2)[C@@H](CC(C)C)C(=O)O1</smiles>

11

$24 \mathrm{~h}, 63 \%$ $>95: 5 \mathrm{dr},>99: 1$ er<smiles>COc1ccc([C@H]2C=C(C(C)=O)OC(=O)[C@@H]2Cc2ccccc2)cc1</smiles>

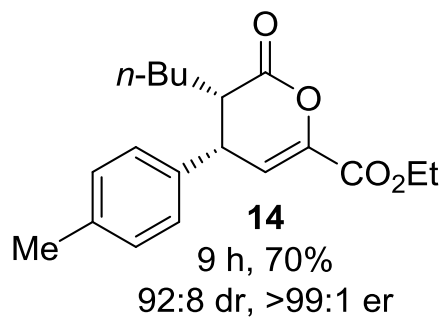<smiles>CCCC[C@@H]1C(=O)OC(OC(C)=O)=C[C@@H]1c1ccc(Br)cc1</smiles>

$89: 11 \mathrm{dr}, 99: 1$ er

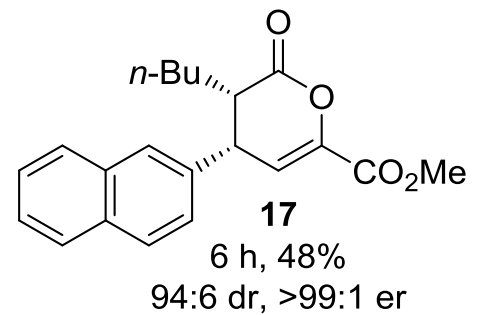

${ }^{a}$ Isolated yields of single diastereoisomers ( $>95: 5 \mathrm{dr}$ ) after column chromatography.

${ }^{b} \mathrm{dr}$ determined by ${ }^{1} \mathrm{H}$ NMR analysis of the crude reaction mixtures.

${ }^{\mathrm{c}}$ er of major diastereoisomer determined by HPLC analysis.

To investigate the reduced diastereoselectivity observed in certain cases, a series of control experiments was performed (Table 3). Treating isolated syn-dihydropyranone 9 ( $>95: 5 \mathrm{dr}$ ) with NHC precatalyst $2(15 \mathrm{~mol} \%)$ and $\mathrm{Et}_{3} \mathrm{~N}(15 \mathrm{~mol} \%)$ resulted in epimerisation, returning 9 in $89: 11 \mathrm{dr}$ after $65 \mathrm{~h}$ at room temperature (Table 3, entry 1). Furthermore, significant amounts of products 18 and 19 arising from double bond isomerisation were also observed, indicating that the initial deprotonation leading to both epimerisation and isomerisation is most likely to

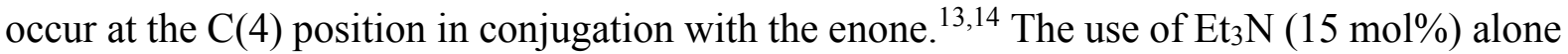
resulted in reduced amounts of both epimerisation (93:7 dr after $65 \mathrm{~h}$ ) and isomerisation, suggesting that the free NHC is capable of deprotonating 9 (Table 3, entry 2). Treating 9 with 
a stoichiometric amount of $\mathrm{Et}_{3} \mathrm{~N}$ (1 eq) resulted in increased levels of isomerisation into both 18 and 19 (Table 3, entry 3), while increased reaction times led to further isomerisation and increased epimerisation of the remaining dihydropyranone 9 (Table 3, entry 4). The NHC and base-promoted epimerisation and isomerisation of the product syn-dihydropyranones could therefore account for the reduced diastereoselectivity and lower yields obtained in certain instances (Table 2).

\section{Table 3}

Investigation into the epimerisation of $s y n-9$

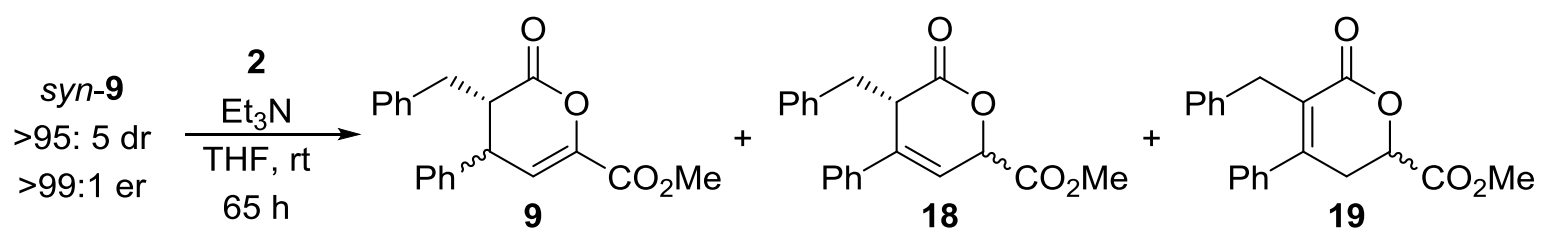

\begin{tabular}{cccccc}
\hline Entry & $\mathbf{2}(\mathrm{mol} \%)$ & $\mathrm{Et}_{3} \mathrm{~N}(\mathrm{~mol} \%)$ & $\mathbf{9 / 1 8} / \mathbf{1 9}^{\mathrm{a}}$ & $\mathrm{dr} \mathrm{9}^{\mathrm{a}}$ & $\mathrm{dr}^{\mathbf{1 8}}$ \\
\hline 1 & 15 & 15 & $50: 30: 20$ & $89: 11$ & $59: 41$ \\
2 & - & 15 & $92: 8: 0$ & $93: 7$ & $55: 45$ \\
3 & - & 100 & $48: 37: 15$ & $94: 6$ & $60: 40$ \\
$4^{\mathrm{b}}$ & - & 100 & $27: 14: 59$ & $80: 20$ & $52: 48$
\end{tabular}

${ }^{a}$ Determined by ${ }^{1} \mathrm{H}$ NMR analysis of the crude reaction mixtures.

${ }^{\mathrm{b}}$ Entry 3 re-subjected to the same conditions for a further $120 \mathrm{~h}$.

\section{$2.3 \quad$ Reactions with $\alpha, \beta$-unsaturated $\gamma$-ketoesters}

Next, the use of $\alpha, \beta$-unsaturated $\gamma$-ketoesters in the NHC-catalysed redox process with $\alpha$ aroyloxyaldehydes was investigated (Table 4). In this case, only $5 \mathrm{~mol} \%$ NHC precatalyst 2 was required alongside $\mathrm{Et}_{3} \mathrm{~N}$ (1.5 eq) in $\mathrm{THF}$ at room temperature to form the corresponding syn-dihydropyranones in high yields and with excellent stereoselectivity. $\alpha$-Aroyloxyaldehydes bearing alkyl and benzyl substituents were well tolerated, forming products 20-22 in high yields with excellent diastereo- and enantioselectivity ( $>95: 5 \mathrm{dr},>99: 1 \mathrm{er}$ ). The relative and absolute configuration of $\mathbf{2 1}$ was confirmed through comparison of its spectroscopic data and specific rotation $\left[>99: 1 \mathrm{er},[\alpha]_{\mathrm{D}}^{20}+173\left(c 1.05\right.\right.$ in $\left.\left.\mathrm{CHCl}_{3}\right)\right]$ with the literature $\left[>99: 1 \mathrm{er},[\alpha]_{\mathrm{D}}^{20}\right.$ $\left.+107\left(c 0.82, \mathrm{CHCl}_{3}\right)\right],{ }^{6 c}$ with all other products in this series assigned by analogy. The reaction using an $\alpha, \beta$-unsaturated $\gamma$-ketoester bearing a methyl substituent needed to be heated at $40{ }^{\circ} \mathrm{C}$ 


\section{Table 4}

Scope of the reaction using $\alpha, \beta$-unsaturated $\gamma$-ketoesters ${ }^{\mathrm{a}-\mathrm{c}}$

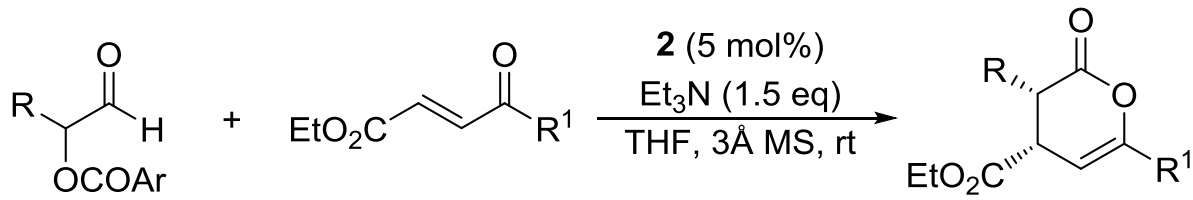

(1.5 eq)

20-29

$\mathrm{Ar}=4-\mathrm{NO}_{2} \mathrm{C}_{6} \mathrm{H}_{4}$

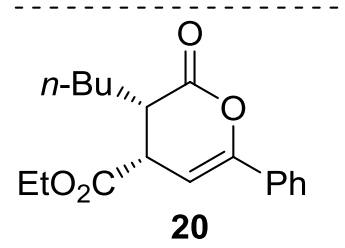

$5 \mathrm{~h}, 85 \%$

$>95: 5 \mathrm{dr},>99: 1 \mathrm{er}$<smiles>CCOC(=O)[C@H]1C=C(C)OC(=O)[C@H]1Cc1ccccc1</smiles>

23

$7 \mathrm{~h}, 65 \%{ }^{\mathrm{e}}$ $>95: 5 \mathrm{dr}, 98.5: 1.5 \mathrm{er}$<smiles>CCOC(=O)[C@H]1C=C(c2ccc(Cl)cc2)OC(=O)[C@H]1C</smiles>

80:20 dr, >99:1 er<smiles>CCOC(=O)[C@H]1C=C(c2ccccc2)OC(=O)[C@H]1Cc1ccccc1</smiles>

2 h, $79 \%^{d}$ $>95: 5 \mathrm{dr},>99: 1 \mathrm{er}$<smiles>CCOC(=O)[C@H]1C=C(c2ccc(C)cc2)OC(=O)[C@H]1C(=O)O[Ga]O[Ge]</smiles><smiles>CCOC(=O)[C@H]1C=C(c2ccc(C(F)(F)F)cc2)OC(=O)[C@H]1Cc1ccccc1</smiles>

$80: 20 \mathrm{dr},>99: 1$ er<smiles>CCOC(=O)[C@H]1C=C(c2ccccc2)OC(=O)[C@H]1CCOBr</smiles>

$9 \mathrm{~h}, 85 \%$ $90: 10 \mathrm{dr},>99: 1$ er<smiles>CCOC(=O)[C@H]1C=C(c2ccc(OC)cc2)OC(=O)[C@H]1[18O][Mg]</smiles><smiles>CCOC(=O)[C@H]1C=C(c2ccc3ccccc3c2)OC(=O)[C@H]1C</smiles>

${ }^{a}$ Isolated yields of single diastereoisomers ( $>95: 5 \mathrm{dr}$ ) after column chromatography.

${ }^{\mathrm{b}} \mathrm{dr}$ determined by ${ }^{1} \mathrm{H}$ NMR analysis of the crude reaction mixtures.

${ }^{c}$ er of major diastereoisomer determined by HPLC analysis.

d 10 mol\% 2 used.

e Reaction performed at $40{ }^{\circ} \mathrm{C}$ using $\mathrm{Cs}_{2} \mathrm{CO}_{3}$ instead of $\mathrm{Et}_{3} \mathrm{~N}$.

and required the use of $\mathrm{Cs}_{2} \mathrm{CO}_{3}$ as base to form syn-dihydropyranone $\mathbf{2 3}$ in $65 \%$ yield as a single diastereoisomer in high enantioselectivity. The absolute configuration of $\mathbf{2 3}$ could also be confirmed through comparison of its specific rotation $\left[98.5: 1.5 \mathrm{er},[\alpha]_{\mathrm{D}}^{20}+271(c 0.32\right.$ in $\left.\left.\mathrm{CHCl}_{3}\right)\right]$ with the literature $\left[>99: 1 \mathrm{er},[\alpha]_{\mathrm{D}}^{20}+251\left(c 0.93, \mathrm{CHCl}_{3}\right)\right] .{ }^{6 \mathrm{c}}$ The use of $\alpha, \beta$-unsaturated $\gamma$-ketoester bearing electron-rich aryl substituents worked particularly well, forming syndihydropyranones $\mathbf{2 4}$ and $\mathbf{2 5}$ in high yield with excellent levels of stereoselectivity ( $>95: 5 \mathrm{dr}$, 
$>99: 1$ er). However, the incorporation of aryl groups containing electron-withdrawing substituents led to the formation of $\mathbf{2 6}$ and $\mathbf{2 7}$ in lower yields with reduced diastereoselectivity (80:20 dr), although the enantioselectivity remained high in both cases ( $>99: 1$ er). A similar reduction in diastereoselectivity was observed by Bode and co-workers in the related NHCcatalysed redox reaction of an $\alpha$-chloroaldehyde with an $\alpha, \beta$-unsaturated $\gamma$-ketoester bearing a 4-bromo substituted aryl ring. ${ }^{6 \mathrm{~b}}$ The reaction of a methyl substituted $\alpha$-aroyloxyaldehyde with a 2-naphthyl substituted $\alpha, \beta$-unsaturated $\gamma$-ketoester under the previously optimised conditions gave the largest reduction in diastereoselectivity, with syn-28 and anti-29 formed as a 50:50 mixture, although both diastereoisomers were obtained in excellent enantioselectivity ( $>99: 1$ er).

The dramatic reduction in diastereoselectivity observed with the 2-naphthyl substituted $\alpha, \beta$ unsaturated $\gamma$-ketoester was probed by treating isolated syn-dihydropyranone $\mathbf{2 8}(>95: 5 \mathrm{dr})$ with $\mathrm{Et}_{3} \mathrm{~N}$ (1.5 eq) in $\mathrm{THF}$ at room temperature (Scheme 3). After 16 hours, the dihydropyranone had epimerized into a 50:50 mixture of diastereoisomers, with both obtained in >99:1 er. This is consistent with the result from the initial NHC-catalysed process, suggesting that the initially formed syn-diastereoisomer is epimerized under the basic reaction conditions. In this case, it is likely that epimerisation occurs at the $\mathrm{C}(4)$ position and the presence of conjugated electron-deficient aryl substituents in the $\mathrm{C}(6)$ position increases the propensity for deprotonation, which accounts for the reduced diastereoselectivity observed in these cases. However, selective epimerisation at the $\mathrm{C}(3)$ position adjacent to the lactone cannot be unambiguously ruled out.

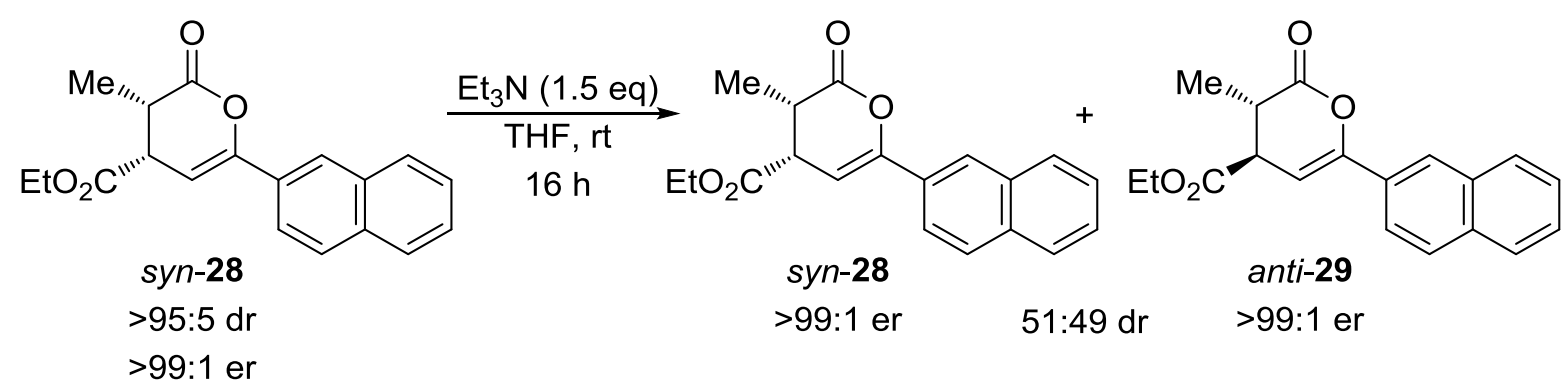

Scheme 3. Base-promoted epimerisation of syn-28

\subsection{Proposed mechanism}

The proposed reaction mechanism is shown in Scheme 4. Initially, the catalytically active free $\mathrm{NHC}$ is generated through deprotonation of NHC precursor 2. Nucleophilic addition of the free NHC 30 into the $\alpha$-aroyloxyaldehyde generates adduct $\mathbf{3 1}$, which is likely to be the 
catalytic resting state. ${ }^{10 \mathrm{~b}}$ Reversible deprotonation of $\mathbf{3 1}$ transiently forms Breslow intermediate 32 that can eliminate 4-nitrobenzoate. The resulting enol $\mathbf{3 3}$ can be deprotonated to form the key azolium enolate intermediate 34, which can undergo an enantioselective asynchronous endo-hetero-Diels-Alder reaction with an appropriate unsaturated ketoester. Finally, elimination from intermediate 35 releases the product syn-dihydropyranone and regenerates the free NHC catalyst. This mechanistic proposal is consistent with the computational studies from Bode and co-workers on related NHC-catalysed [4+2]-heteroDiels-Alder processes. ${ }^{15}$

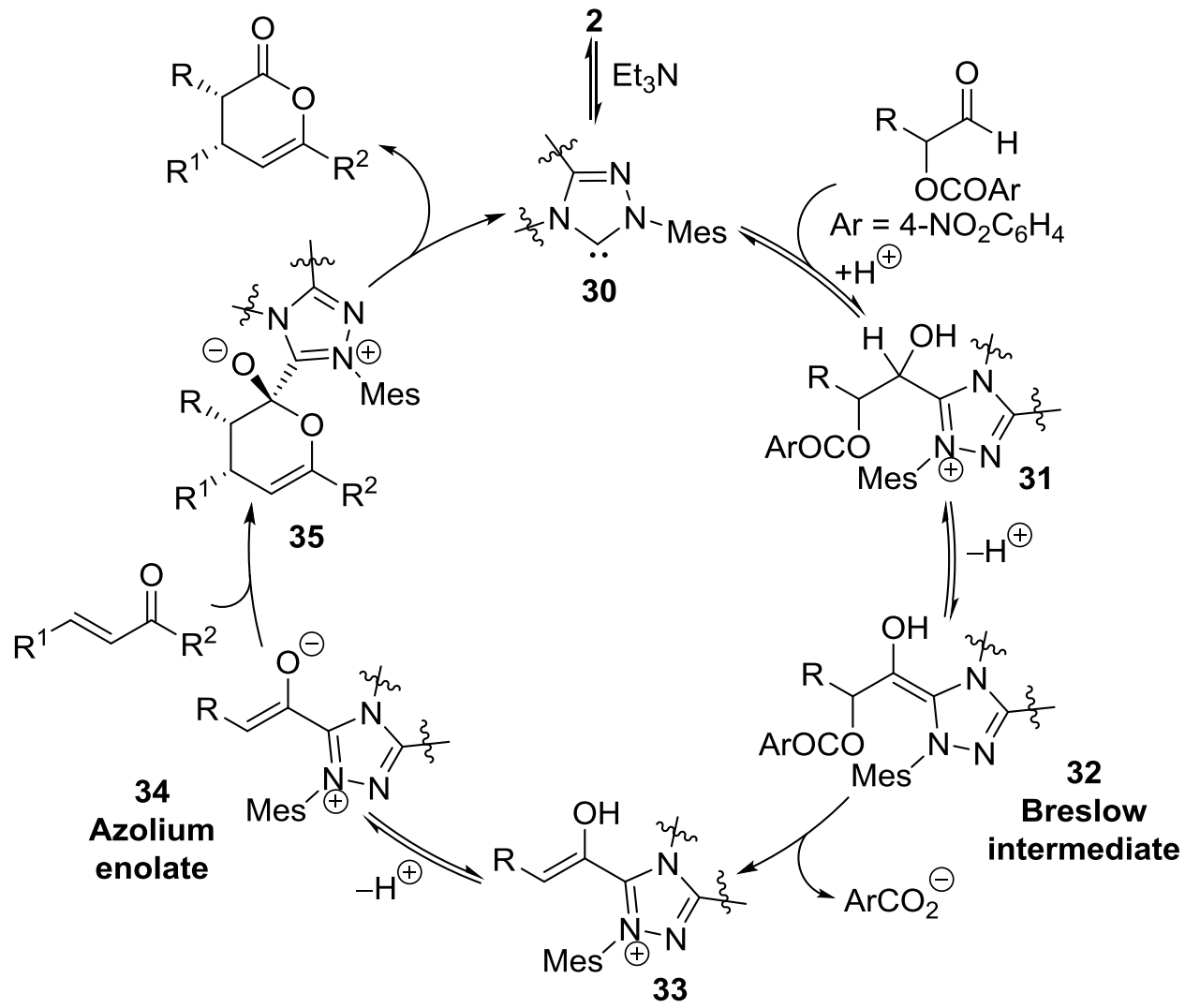

Scheme 4. Proposed reaction mechanism

\section{Conclusion}

$\alpha$-Aroyloxyaldehydes are efficient azolium enolate precursors in enantioselective NHCcatalysed [4+2] hetero-Diels-Alder reactions with unsaturated ketoesters. Reactions using either $\beta, \gamma$-unsaturated $\alpha$-ketoesters or $\alpha, \beta$-unsaturated $\gamma$-ketoesters form the corresponding syndihydropyranone products with generally high diastereoselectivity and excellent enantioselectivity. In cases where the unsaturated ketoester bears an electron-deficient aryl substituent, the initially-formed syn-dihydropyranones are more susceptible to epimerisation at 
the $\mathrm{C}(4)$-position under the reaction conditions, which lowers the observed diastereoselectivity of the products.

\section{Experimental}

\subsection{General}

Anhydrous reactions were carried out in flame-dried glassware under an inert atmosphere $\left(\mathrm{N}_{2}\right.$ or Ar) using standard vacuum line techniques. Anhydrous THF and $\mathrm{Et}_{2} \mathrm{O}$ were obtained after passing through an alumina column (Mbraun SPS-800). Petrol is defined as petroleum ether $40-60{ }^{\circ} \mathrm{C}$. All other solvents and commercial reagents were used as received without further purification. $\alpha$-Aroyloxyaldehydes were prepared according to previously reported procedures. $^{10}$

Room temperature (rt) refers to $20-25^{\circ} \mathrm{C}$ and a temperature of $0{ }^{\circ} \mathrm{C}$ was obtained using an ice/water bath. Reaction involving heating were performed using DrySyn blocks and a contact thermocouple. Under reduced pressure refers to the use of a rotary evaporator with vacuum controller.

Analytical thin layer chromatography was performed on pre-coated aluminium plates (Kieselgel $60 \mathrm{~F}_{254}$ silica) and visualisation was achieved using ultraviolet light $(254 \mathrm{~nm})$ and/or staining with a $1 \%$ aqueous $\mathrm{KMnO}_{4}$ solution. Flash column chromatography was performed on Kieselgel 60 silica in the solvent system stated.

Melting points were recorded on an Electrothermal 9100 melting point apparatus, (dec) refers to decomposition. Optical rotations were measured on a Perkin Elmer Precisely/Model-341 polarimeter operating at the sodium $\mathrm{D}$ line with a $100 \mathrm{~mm}$ path cell at $20^{\circ} \mathrm{C}$.

HPLC analyses were obtained on either a Gilson HPLC consisting of a Gilson 305 pump, Gilson 306 pump, Gilson 811C dynamic mixer, Gilson 805 manometric module, Gilson 401C dilutor, Gilson 213XL sample injector and sample detection was performed with a Gilson 118 UV/vis detector or a Shimadzu HPLC consisting of a DGU-20A5 degasser, LC-20AT liquid chromatography SIL-20AHT autosampler, CMB-20A communications bus module, SPDM20A diode array detector and a CTO-20A column oven. Separation was achieved using Chiralcel OD-H and OJ-H columns or a Chiralpak AD-H column. All HPLC traces were compared with an authentic racemic spectrum prepared in analogous fashion using ( \pm )-2.

Infrared spectra $\left(v_{\max }\right)$ were recorded on a Shimadzu IRAffinity-1 Fourier transform IR spectrophotometer using either thin film or solid using Pike MIRacle ATR accessory. 
${ }^{1} \mathrm{H},{ }^{13} \mathrm{C}\left\{{ }^{1} \mathrm{H}\right\}$, and ${ }^{19} \mathrm{~F}\left\{{ }^{1} \mathrm{H}\right\}$ NMR spectra were acquired on either a Bruker Avance $300\left({ }^{1} \mathrm{H}\right.$ $\left.300 \mathrm{MHz} ;{ }^{13} \mathrm{C}\left\{{ }^{1} \mathrm{H}\right\} 75 \mathrm{MHz} ;{ }^{19} \mathrm{~F}\left\{{ }^{1} \mathrm{H}\right\} 282 \mathrm{MHz}\right)$, a Bruker Avance II $400\left({ }^{1} \mathrm{H} 400 \mathrm{MHz}\right.$; $\left.{ }^{13} \mathrm{C}\left\{{ }^{1} \mathrm{H}\right\} 100 \mathrm{MHz} ;{ }^{19} \mathrm{~F}\left\{{ }^{1} \mathrm{H}\right\} 376 \mathrm{MHz}\right)$, or a Bruker Ultrashield $500\left({ }^{1} \mathrm{H} 500 \mathrm{MHz}\right)$ spectrometer at room temperature in the deuterated solvent stated. All chemical shifts are quoted in parts per million (ppm) relative to the residual solvent peak. All coupling constants, $J$, are quoted in Hz. Multiplicities are indicated as s (singlet), d (doublet), t (triplet), q (quartet), m (multiplet), and multiples thereof. The abbreviation Ar denotes aromatic and app denotes apparent.

High resolution mass spectrometry $(\mathrm{m} / \mathrm{z})$ data was acquired by electrospray ionisation $(\mathrm{ESI})$, atmospheric pressure chemical ionisation (APCI) or nanospray ionisation (NSI) at the EPSRC UK National Mass Spectrometry Facility at Swansea University.

\subsection{General procedure for the synthesis of $\beta, \gamma$-unsaturated $\alpha$-ketoesters}

Part 1: Based upon a literature procedure procedure, ${ }^{16} \mathrm{KOH}(1.5 \mathrm{eq})$ in $\mathrm{MeOH}(6 \mathrm{M})$ was added to a solution of pyruvic acid (1.0 eq), the required aryl aldehyde (1.0 eq) and $\mathrm{MeOH}$ (12 M) at $0{ }^{\circ} \mathrm{C}$. The first equivalent of $\mathrm{KOH}$ solution was added dropwise over $30 \mathrm{~min}$, the rest was added as one portion. The reaction mixture was heated at $40{ }^{\circ} \mathrm{C}$ for $1 \mathrm{~h}$ before being cooled to $0{ }^{\circ} \mathrm{C}$ and stirred overnight. The precipitate was collected by filtration and washed with cold $\mathrm{MeOH}(\times 2)$ and $\mathrm{Et}_{2} \mathrm{O}$ before being dried under vacuum to give the desired $\beta, \gamma$-unsaturated $\alpha$ ketoacid potassium salt.

Part 2: Based upon a literature procedure procedure, ${ }^{17}$ acetyl chloride (11.5 eq) was added dropwise to the appropriate alcohol $(0.2 \mathrm{M})$ at $0{ }^{\circ} \mathrm{C}$ before the required potassium salt $(1.0 \mathrm{eq})$ was added. The reaction mixture was warmed to $\mathrm{rt}$ and stirred for $2 \mathrm{~h}$ before being heated at reflux for $6 \mathrm{~h}$. The solution was cooled to rt, concentrated under reduced pressure and then partitioned between $\mathrm{CH}_{2} \mathrm{Cl}_{2}$ and $\mathrm{H}_{2} \mathrm{O}$. The layers were separated and the aqueous layer extracted with $\mathrm{CH}_{2} \mathrm{Cl}_{2}(\times 2)$. The combined organic phases were washed with a saturated $\mathrm{NaHCO}_{3}$ solution and $\mathrm{H}_{2} \mathrm{O}$ before being dried over $\mathrm{Na}_{2} \mathrm{SO}_{4}$, filtered and concentrated to give the crude $\beta, \gamma$-unsaturated $\alpha$-ketoester that was purified by column chromatography.

\subsection{1 (E)-Methyl 2-oxo-4-phenylbut-3-enoate 8}

Following General Procedure 4.2 (part 1), KOH (16.8 g, $300 \mathrm{mmol})$ in $\mathrm{MeOH}(60 \mathrm{~mL})$, pyruvic acid (13.8 mL, $200 \mathrm{mmol})$ and benzaldehyde $(20.4 \mathrm{~mL}, 200 \mathrm{mmol})$ in $\mathrm{MeOH}(15 \mathrm{~mL})$ were reacted to give potassium $(E)$-2-oxo-4-phenylbut-3-enoate $(39.0 \mathrm{~g}, 91 \%)$ as a yellow solid with spectroscopic data in accordance with the literature. ${ }^{18} \mathrm{mp} 246-248{ }^{\circ} \mathrm{C}\left\{\right.$ Lit. $\left.{ }^{18} 248{ }^{\circ} \mathrm{C}\right\} ;{ }^{1} \mathrm{H}$ 
NMR (400 MHz, D $2 \mathrm{O}) \delta_{\mathrm{H}}: 6.78(1 \mathrm{H}, \mathrm{d}, J 15.0, \mathrm{PhCH}=\mathrm{CH}), 7.34-7.42(3 \mathrm{H}, \mathrm{m}, \operatorname{Ar}(3,4,5) H)$, $7.39(1 \mathrm{H}, \mathrm{d}, J 15.0, \mathrm{C} \mathrm{PhCH}=\mathrm{CH}), 7.57-7.61(2 \mathrm{H}, \mathrm{m}, \operatorname{Ar}(2,6) H)$.

Following General Procedure 4.2 (part 2), potassium (E)-2-oxo-4-phenylbut-3-enoate (7.48 g, $35 \mathrm{mmol})$ and acetyl chloride $(28.6 \mathrm{~mL}, 402 \mathrm{mmol})$ in $\mathrm{MeOH}(200 \mathrm{~mL})$ were reacted. The crude was purified by column chromatography (90:10 petrol : EtOAc), then recrystallised from $\mathrm{MeOH}$ to give $8(1.16 \mathrm{~g}, 17 \%)$ as a yellow crystalline solid with spectroscopic data in accordance with the literature. ${ }^{18} \mathrm{mp} 69-71{ }^{\circ} \mathrm{C}\left\{\right.$ Lit. $\left.^{18} 69-70{ }^{\circ} \mathrm{C}\right\} ;{ }^{1} \mathrm{H}$ NMR $\left(300 \mathrm{MHz}, \mathrm{CDCl}_{3}\right)$ $\delta_{\mathrm{H}}: 3.94\left(3 \mathrm{H}, \mathrm{s}, \mathrm{OCH}_{3}\right), 7.38(1 \mathrm{H}, \mathrm{d}, J 16.1, H \mathrm{C}=\mathrm{C}), 7.42-7.49(3 \mathrm{H}, \mathrm{m}, \mathrm{Ar} H), 7.61-7.67(2 \mathrm{H}$, m, $\operatorname{Ar} H), 7.89(1 \mathrm{H}, \mathrm{d}, J 16.2, H \mathrm{C}=\mathrm{C})$.

\subsection{2 (E)-Ethyl 2-oxo-4-(p-tolyl)but-3-enoate 36}

Following General Procedure 4.2 (part 1), $\mathrm{KOH}(1.40 \mathrm{~g}, 24.9 \mathrm{mmol})$ in $\mathrm{MeOH}(5 \mathrm{~mL})$, pyruvic acid $(1.15 \mathrm{~mL}, 16.6 \mathrm{mmol})$ and $p$-tolualdehyde $(1.96 \mathrm{~mL}, 16.6 \mathrm{mmol})$ in $\mathrm{MeOH}(2$ $\mathrm{mL})$ were reacted to give potassium $(E)$-2-oxo-4-(p-tolyl)but-3-enoate (2.78 g, $12.2 \mathrm{mmol}$, $73 \%$ ) as yellow solid, with spectroscopic data in accordance with the literature. ${ }^{18} \mathrm{mp} 242-244$ ${ }^{\circ} \mathrm{C}(\mathrm{dec}) ;{ }^{1} \mathrm{H}$ NMR $\left(400 \mathrm{MHz}, \mathrm{D}_{2} \mathrm{O}\right) \delta_{\mathrm{H}}: 2.28(3 \mathrm{H}, \mathrm{s}, \mathrm{ArCH}), 6.74(1 \mathrm{H}, \mathrm{d}, J 16.4, \mathrm{ArCH}=\mathrm{CH})$, $7.23(2 \mathrm{H}, \mathrm{d}, J$ 8.1, $\mathrm{ArH}), 7.51(2 \mathrm{H}, \mathrm{d}, J$ 8.2, $\mathrm{ArH}), 7.59$ (1H, d, $J 16.4, \mathrm{ArCH}=\mathrm{CH})$.

Following General Procedure 4.2 (part 2), potassium (E)-2-oxo-4-( $p$-tolyl)but-3-enoate (1.00 g, $4.38 \mathrm{mmol})$ and acetyl chloride $(3.58 \mathrm{~mL}, 50.4 \mathrm{mmol})$ in ethanol $(30 \mathrm{~mL})$ were reacted. The crude was purified by column chromatography (95:5 petrol : EtOAc) to give $\mathbf{3 6}(0.560 \mathrm{~g}, 58 \%)$ as a yellow solid, with spectroscopic data in accordance with the literature. ${ }^{17} \mathrm{mp} 40-42{ }^{\circ} \mathrm{C}$ $\left\{\right.$ Lit. $\left.{ }^{17} 47-48{ }^{\circ} \mathrm{C}\right\} ;{ }^{1} \mathrm{H}$ NMR $\left(300 \mathrm{MHz}, \mathrm{CDCl}_{3}\right) \delta_{\mathrm{H}}: 1.41\left(3 \mathrm{H}, \mathrm{t}, J 7.1, \mathrm{OCH}_{2} \mathrm{CH}_{3}\right), 2.40(3 \mathrm{H}, \mathrm{s}$, $\left.\mathrm{ArCH}_{3}\right), 4.39\left(2 \mathrm{H}, \mathrm{q}, J\right.$ 7.1, $\left.\mathrm{OCH}_{2} \mathrm{CH}_{3}\right), 7.23(2 \mathrm{H}, \mathrm{d}, J 7.9, \mathrm{ArH}), 7.32(1 \mathrm{H}, \mathrm{d}, J$ 16.1, $\mathrm{ArCH}=\mathrm{CH}), 7.53(2 \mathrm{H}, \mathrm{d}, J$ 8.2, $\mathrm{ArH}), 7.84(1 \mathrm{H}, \mathrm{d}, J 16.1, \mathrm{ArCH}=\mathrm{CH})$.

\subsection{3 (E)-Methyl 4-(4-methoxyphenyl)-2-oxobut-3-enoate 37}

Following General Procedure 4.2 (part 1), KOH (1.11 g, $19.8 \mathrm{mmol}$ ) in $\mathrm{MeOH}(4 \mathrm{~mL})$, pyruvic acid $(0.917 \mathrm{~mL}, 13.2 \mathrm{mmol})$ and p-anisaldehyde $(1.80 \mathrm{~g}, 1.61 \mathrm{~mL}, 13.2 \mathrm{mmol})$ in $\mathrm{MeOH}(1.5 \mathrm{~mL})$ were reacted to give potassium $(E)$-4-(4-methoxyphenyl)-2-oxobut-3-enoate $(2.31 \mathrm{~g}, 72 \%)$ as a yellow solid, with spectroscopic data in accordance with the literature. ${ }^{16} \mathrm{mp}$ 250-252 ${ }^{\circ} \mathrm{C}\left\{\right.$ Lit. $\left.^{16} 248{ }^{\circ} \mathrm{C}\right\} ;{ }^{1} \mathrm{H}$ NMR $\left(400 \mathrm{MHz}, \mathrm{D}_{2} \mathrm{O}\right) \delta_{\mathrm{H}}: 6.66(1 \mathrm{H}, \mathrm{d}, J 16.4, \mathrm{CH}=\mathrm{CH}), 6.95$ (2H, d, J 8.8, $\mathrm{ArH}), 7.50-7.69$ (3H, m, $\mathrm{Ar} H$ and $\mathrm{CH}=\mathrm{CH})$.

Following General Procedure 4.2 (part 2), potassium (E)-2-oxo-4-(4-methoxyphenyl)but-3enoate $(2.00 \mathrm{~g}, 8.19 \mathrm{mmol})$ and acetyl chloride $(6.70 \mathrm{~mL}, 94.2 \mathrm{mmol})$ in $\mathrm{MeOH}(60 \mathrm{~mL})$ were 
reacted. The crude was purified by column chromatography (85:15 petrol : EtOAc) to give 37 $(1.37 \mathrm{~g}, 76 \%)$ as a yellow crystalline solid, with spectroscopic data in accordance with the literature. ${ }^{18} \mathrm{mp} 95-96{ }^{\circ} \mathrm{C}\left\{\right.$ Lit. $\left.^{18} 99-100{ }^{\circ} \mathrm{C}\right\} ;{ }^{1} \mathrm{H}$ NMR $\left(300 \mathrm{MHz}, \mathrm{CDCl}_{3}\right) \delta_{\mathrm{H}}: 3.80(3 \mathrm{H}, \mathrm{s}$, $\left.\mathrm{OCH}_{3}\right), 3.86\left(3 \mathrm{H}, \mathrm{s}, \mathrm{OCH}_{3}\right), 6.87(2 \mathrm{H}, \mathrm{d}, J$ 8.8, $\mathrm{ArH}), 7.19(1 \mathrm{H}, \mathrm{d}, J 16.0, \mathrm{ArCH}=\mathrm{CH}), 7.54$ $(2 \mathrm{H}, \mathrm{d}, J 8.5, \mathrm{Ar} H), 7.79(1 \mathrm{H}, \mathrm{d}, J 16.0, \mathrm{ArCH}=\mathrm{CH})$.

\subsection{4 (E)-Methyl 4-(4-fluorophenyl)-2-oxobut-3-enoate 38}

Following General Procedure 4.2 (part 1), $\mathrm{KOH}(1.36 \mathrm{~g}, 24.2 \mathrm{mmol})$ in $\mathrm{MeOH}(6 \mathrm{~mL})$, pyruvic acid $(1.12 \mathrm{~mL}, 16.1 \mathrm{mmol})$ and 4-fluorobenzaldehyde $(2.00 \mathrm{~g}, 1.73 \mathrm{~mL}, 16.1 \mathrm{mmol})$ in $\mathrm{MeOH}(2 \mathrm{~mL})$ were reacted to give potassium $(E)$-4-(4-fluorophenyl)-2-oxobut-3-enoate $(2.67 \mathrm{~g}, 71 \%)$ as a yellow solid, with spectroscopic data in accordance with the literature. ${ }^{19} \mathrm{mp}$ 205-208 ${ }^{\circ} \mathrm{C}(\mathrm{dec}) ;{ }^{1} \mathrm{H}$ NMR $\left(500 \mathrm{MHz}, \mathrm{D}_{2} \mathrm{O}\right) \delta_{\mathrm{H}}: 6.73(1 \mathrm{H}, \mathrm{d}, J 16.3, \mathrm{ArCH}=\mathrm{CH}), 7.11(2 \mathrm{H}, \mathrm{t}$, $J$ 8.2, $\operatorname{ArC}(2) H), 7.59(1 \mathrm{H}, \mathrm{d}, J 16.4, \operatorname{ArCH}=\mathrm{CH}), 7.64(2 \mathrm{H}, \mathrm{dd}, J 7.4,5.2, \operatorname{ArC}(3) H) ;{ }^{19} \mathrm{~F}$ NMR $\left(376 \mathrm{MHz}, \mathrm{D}_{2} \mathrm{O}\right) \delta_{\mathrm{F}}:-109.0(\mathrm{ArC}(4) F)$.

Following General Procedure 4.2 (part 2), potassium (E)-4-(4-fluorophenyl)-2-oxobut-3enoate $(1.00 \mathrm{~g}, 4.31 \mathrm{mmol})$ and acetyl chloride $(3.53 \mathrm{~mL}, 49.6 \mathrm{mmol})$ in $\mathrm{MeOH}(40 \mathrm{~mL})$ were reacted. The crude was purified by column chromatography (95:5 petrol : EtOAc) to give $(E)$ methyl 4-(4-fluorophenyl)-2-oxobut-3-enoate ( $0.320 \mathrm{~g}, 36 \%)$ as a yellow crystalline solid, with spectroscopic data in accordance with the literature. ${ }^{20} \mathrm{mp} 86-87^{\circ} \mathrm{C} ; v_{\max }$ (solid) $1722(\mathrm{C}=\mathrm{O})$, $1690(\mathrm{C}=\mathrm{O}) ;{ }^{1} \mathrm{H} \mathrm{NMR}\left(400 \mathrm{MHz}, \mathrm{CDCl}_{3}\right) \delta_{\mathrm{H}}: 3.87\left(3 \mathrm{H}, \mathrm{s}, \mathrm{OCH}_{3}\right), 7.06(2 \mathrm{H}, \mathrm{t}, J 8.6, \operatorname{ArC}(3) H)$, $7.25(1 \mathrm{H}, \mathrm{dd}, J 16.2,0.6, \mathrm{CH}=\mathrm{C} H), 7.58(2 \mathrm{H}, \mathrm{dd}, J$ 8.7, 5.4, $\operatorname{ArC}(2) H), 7.78(1 \mathrm{H}, \mathrm{d}, J 16.1$, $\mathrm{C} H=\mathrm{CH}) ;{ }^{19} \mathrm{~F}$ NMR $\left(376 \mathrm{MHz}, \mathrm{CDCl}_{3}\right) \delta_{\mathrm{F}}:-107.3\left(\mathrm{tt}, J\right.$ 8.1, 5.5, C(4)F); ${ }^{13} \mathrm{C}\left\{{ }^{1} \mathrm{H}\right\} \mathrm{NMR}(101$ $\left.\mathrm{MHz}, \mathrm{CDCl}_{3}\right) \delta_{\mathrm{C}}: 53.1\left(\mathrm{OCH}_{3}\right), 116.4(\mathrm{~d}, J 22.0, C(3)), 120.1(\mathrm{~d}, J 2.2, \mathrm{ArCH}=C \mathrm{H}), 130.3(\mathrm{~d}$, $J$ 3.1, C(1)), $131.2\left(\mathrm{~d}, J\right.$ 8.8, C(2)), $147.2(\mathrm{ArCH}=\mathrm{CH}), 162.5\left(\mathrm{CO}_{2}\right), 164.7(\mathrm{~d}, J 253.9, C(4))$, 182.1 $(\mathrm{CH}=\mathrm{CHCO}) ; \mathrm{HRMS}\left(\mathrm{NSI}^{+}\right) \mathrm{C}_{11} \mathrm{H}_{10} \mathrm{O}_{3} \mathrm{~F}[\mathrm{M}+\mathrm{H}]^{+}$found 209.0610, requires 209.0608 $(+0.7 \mathrm{ppm})$.

\subsection{5 (E)-Methyl 4-(4-bromophenyl)-2-oxobut-3-enoate 39}

Following General Procedure 4.2 (part 1), KOH (4.55 g, $81.1 \mathrm{mmol}$ ) in $\mathrm{MeOH}(15 \mathrm{~mL})$, pyruvic acid $(3.81 \mathrm{~mL}, 54.1 \mathrm{mmol})$ and 4-bromobenzaldehyde $(10.0 \mathrm{~g}, 54.1 \mathrm{mmol})$ in $\mathrm{MeOH}$ $(15 \mathrm{~mL})$ were reacted to give potassium $(E)$-2-oxo-4-(4-bromophenyl)but-3-enoate (15.9 g, $84 \%$ ) as a yellow solid, with spectroscopic data in accordance with the literature. ${ }^{18} \mathrm{mp} 240{ }^{\circ} \mathrm{C}$ (dec) $\left\{\right.$ Lit. $\left.^{18} 233{ }^{\circ} \mathrm{C}\right\} ;{ }^{1} \mathrm{H}$ NMR $\left(400 \mathrm{MHz}, \mathrm{D}_{2} \mathrm{O}\right) \delta_{\mathrm{H}}: 6.91(1 \mathrm{H}, \mathrm{d}, J 17.1, \mathrm{C}(3) H), 7.59-7.60$ (2H, m, $\operatorname{Ar}(3,5) H), 7.62(1 \mathrm{H}, \mathrm{d}, J 17.1, \mathrm{C}(4) H), 7.65-7.68(2 \mathrm{H}, \mathrm{m}, \operatorname{Ar}(2,6) H)$. 
Following General Procedure 4.2 (part 2), potassium (E)-2-oxo-4-(4-bromophenyl)but-3enoate $(2.00 \mathrm{~g}, 6.82 \mathrm{mmol})$ and acetyl chloride $(5.57 \mathrm{~mL}, 78.4 \mathrm{mmol})$ in $\mathrm{MeOH}(40 \mathrm{~mL})$ were reacted. The crude was purified by column chromatography (95:5 petrol : EtOAc) to give 39 $(1.21 \mathrm{~g}, 66 \%)$ as a yellow crystalline solid, with spectroscopic data in accordance with the literature. ${ }^{18} \mathrm{mp} 109-112{ }^{\circ} \mathrm{C}\left\{\right.$ Lit. $\left.^{18} 120{ }^{\circ} \mathrm{C}\right\} ;{ }^{1} \mathrm{H}$ NMR $\left(400 \mathrm{MHz}, \mathrm{CDCl}_{3}\right) \delta_{\mathrm{H}}: 3.87(3 \mathrm{H}, \mathrm{s}$, $\left.\mathrm{OCH}_{3}\right), 7.31(1 \mathrm{H}, \mathrm{d}, J 16.1, \mathrm{ArCH}=\mathrm{CH}), 7.43(2 \mathrm{H}, \mathrm{d}, J 8.5, \mathrm{ArH}), 7.50(2 \mathrm{H}, \mathrm{d}, J 8.5, \mathrm{ArH})$, $7.74(1 \mathrm{H}, \mathrm{d}, J 16.1, \mathrm{ArCH}=\mathrm{CH})$.

\subsection{6 (E)-Methyl 4-(naphthalen-2-yl)-2-oxobut-3-enoate 40}

Following General Procedure 4.2 (part 1), KOH (1.08 g, $19.2 \mathrm{mmol})$ in $\mathrm{MeOH}(3 \mathrm{~mL})$, pyruvic acid $(0.890 \mathrm{~mL}, 12.8 \mathrm{mmol})$ and 2-naphthaldehyde $(2.00 \mathrm{~g}, 12.8 \mathrm{mmol})$ in $\mathrm{MeOH}(3$ $\mathrm{mL}$ ) were reacted to give potassium $(E)$-4-(naphthalen-2-yl)-2-oxobut-3-enoate $(2.46 \mathrm{~g}, 73 \%)$

as a yellow solid, with spectroscopic data in accordance with the literature. ${ }^{18} \mathrm{mp}>320{ }^{\circ} \mathrm{C}$ $\left\{\right.$ Lit. $\left.^{18} 270-272{ }^{\circ} \mathrm{C}(\mathrm{dec})\right\} ;{ }^{1} \mathrm{H}$ NMR $\left(400 \mathrm{MHz}, \mathrm{D}_{2} \mathrm{O}\right) \delta_{\mathrm{H}}: 6.85(1 \mathrm{H}, \mathrm{d}, J 16.5, \mathrm{ArCH}=\mathrm{CH}), 7.50$ $(2 \mathrm{H}, \mathrm{s}, \mathrm{Ar} H), 7.65-7.74(2 \mathrm{H}, \mathrm{m}, \mathrm{Ar} H$, and $\mathrm{ArCH}=\mathrm{CH}), 7.81-7.86(3 \mathrm{H}, \mathrm{m}, \mathrm{ArH}), 7.99(1 \mathrm{H}, \mathrm{s}$, $\operatorname{Ar}(1) H)$.

Following General Procedure 4.2 (part 2), potassium (E)-4-(naphthalen-2-yl)-2-oxobut-3enoate (1.00 g, $3.78 \mathrm{mmol})$ and acetyl chloride (3.08 mL, $43.5 \mathrm{mmol})$ in $\mathrm{MeOH}(30 \mathrm{~mL})$ were reacted. The crude was purified by column chromatography (95:5 petrol : EtOAc) to give $\mathbf{4 0}$ $(0.570 \mathrm{~g}, 63 \%)$ as a yellow solid, with spectroscopic data in accordance with the literature. ${ }^{18}$ mp 94-96 ${ }^{\circ} \mathrm{C}\left\{\right.$ Lit. $\left.^{18}{ }^{70-72}{ }^{\circ} \mathrm{C}\right\} ;{ }^{1} \mathrm{H}$ NMR $\left(400 \mathrm{MHz}, \mathrm{CDCl}_{3}\right) \delta_{\mathrm{H}}: 3.96\left(3 \mathrm{H}, \mathrm{s}, \mathrm{OCH}_{3}\right), 7.49$ $(1 \mathrm{H}, \mathrm{d}, J 16.1, \mathrm{ArCH}=\mathrm{CH}), 7.52-7.61(2 \mathrm{H}, \mathrm{m}, \mathrm{ArH}), 7.77(1 \mathrm{H}, \mathrm{dd}, J$ 8.6, 1.8, $\mathrm{ArH}), 7.84-7.93$ $(3 \mathrm{H}, \mathrm{m}, \mathrm{Ar} H), 8.02-8.09$ (2H, m, $\mathrm{ArCH}=\mathrm{CH}$ and $\mathrm{Ar} H)$.

\subsection{General procedure for the NHC-catalysed redox hetero-Diels-Alder reaction with $\beta, \gamma$-unsaturated $\alpha$-ketoesters}

The appropriate $\alpha$-aroyloxyaldehyde (1.5 eq), $\beta, \gamma$-unsaturated $\alpha$-ketoester (1.0 eq) and NHC precatalyst $2(10 \mathrm{~mol} \%)$ were dissolved in anhydrous THF $(0.075 \mathrm{M})$ in a sealed vial containing $3 \AA$ molecular sieves. The solution was cooled to $0{ }^{\circ} \mathrm{C}$ before $\mathrm{Et}_{3} \mathrm{~N}$ (1.5 eq.) was added. The reaction stirred, allowing to warm to rt, until complete by TLC analysis. The mixture was diluted with EtOAc and washed successively with $1 \mathrm{M} \mathrm{HCl}$, saturated $\mathrm{NaHCO}_{3}$ and brine. The organic layer was dried with $\mathrm{MgSO}_{4}$, filtered and concentrated under reduced pressure to give the crude product, which was purified by column chromatography. 


\subsection{1 (3S,4S)-Methyl 3-benzyl-2-oxo-4-phenyl-3,4-dihydro-2H-pyran-6-carboxylate 9}

Following General Procedure 4.3, 1-oxo-3-phenylpropan-2-yl 4-nitrobenzoate 7 (50 mg, 0.17 mmol), (E)-methyl 2-oxo-4-phenylbut-3-enoate $8(21.0 \mathrm{mg}, 0.11 \mathrm{mmol})$, NHC precatalyst 2 (4.0 mg, $11 \mu \mathrm{mol}), \mathrm{Et}_{3} \mathrm{~N}(23 \mu \mathrm{L}, 0.17 \mathrm{mmol})$ and THF ( $\left.3 \mathrm{~mL}\right)$ were reacted for $12 \mathrm{~h}$. The crude ( $>95: 5 \mathrm{dr}$ ) was purified by column chromatography (90:10 petrol : EtOAc) to give 9 (51 $\mathrm{mg}$, $93 \%$ ) as a colourless solid. mp $106-107^{\circ} \mathrm{C} ;[\alpha]_{\mathrm{D}}^{20}+398\left(c 0.23, \mathrm{CHCl}_{3}\right)$; Chiral HPLC analysis, Chiralpak AD-H (90:10 hexane : IPA, flow rate $\left.1.0 \mathrm{mLmin}^{-1}, 254 \mathrm{~nm}, 30{ }^{\circ} \mathrm{C}\right) \mathrm{t}_{\mathrm{R}}(R, R) 18.0$ $\min , \mathrm{t}_{\mathrm{R}}(S, S) 27.8 \mathrm{~min},>99: 1 \mathrm{er} ; v_{\max }\left(\mathrm{KBr}, \mathrm{cm}^{-1}\right)$ 2956, 2925, 1765, 1628, 1664, 1602, 1495, 1435, 1316, 1100; ${ }^{1} \mathrm{H}$ NMR $\left(400 \mathrm{MHz}, \mathrm{CDCl}_{3}\right) \delta_{\mathrm{H}}: 2.33\left(1 \mathrm{H}, \mathrm{dd}, J 10.6,15.8, \mathrm{C}(3) \mathrm{CH}^{\mathrm{A}} \mathrm{H}^{\mathrm{B}}\right)$, 3.16-3.23 (2H, m, C(3) $\mathrm{CH}^{\mathrm{A}} H^{\mathrm{B}}$ and $\left.\mathrm{C}(3) H\right), 3.55(1 \mathrm{H}, \mathrm{t}, J 6.8, \mathrm{C}(4) H), 3.77\left(3 \mathrm{H}, \mathrm{OCH}_{3}\right), 6.58$ $(1 \mathrm{H}, \mathrm{d}, J$ 6.7, C(5)H), 6.93-7.00 (4H, m, C(3)ArH), 7.15-7.26 (6H, m, C(3)ArH and $\left.\mathrm{C}(3) \mathrm{CH}_{2} \mathrm{Ar} H\right) ;{ }^{13} \mathrm{C}\left\{{ }^{1} \mathrm{H}\right\}$ NMR $\left(75 \mathrm{MHz}, \mathrm{CDCl}_{3}\right) \delta_{\mathrm{C}}: 32.1\left(\mathrm{C}(3) \mathrm{CH}_{2}\right), 40.6(\mathrm{C}(3) H), 44.8$ $(\mathrm{C}(4) H), 52.7\left(\mathrm{OCH}_{3}\right), 118.8$ (C(5)), 126.8 (C(3) $\left.\mathrm{CH}_{2} \mathrm{ArC}(4)\right), 128.4$ (C(4)ArC(4)), 128.4 ( $\mathrm{ArC}), 128.7$ ( $\mathrm{ArC}), 128.9(\mathrm{ArC}), 129.2$ (ArC), 135.8 (CPh-1), $138.1\left(\mathrm{C}(3) \mathrm{CH}_{2} \mathrm{ArC}(1)\right), 141.9$ $(C(6)), 161.0\left(\mathrm{CO}_{2} \mathrm{Me}\right), 168.5(C(2))$; HRMS (ESI+) $\mathrm{C}_{20} \mathrm{H}_{22} \mathrm{O}_{4} \mathrm{~N}\left[\mathrm{M}+\mathrm{NH}_{4}\right]^{+}$found 340.1544 , requires $340.1543(+0.2 \mathrm{ppm})$.

\subsection{2 (3S,4S)-Methyl 3-butyl-2-oxo-4-phenyl-3,4-dihydro-2H-pyran-6-carboxylate 10}

Following General Procedure 4.3, 1-oxohexan-2-yl 4-nitrobenzoate (50 mg, $0.19 \mathrm{mmol}),(E)$ methyl 2-oxo-4-phenylbut-3-enoate 8 (25.2 mg, $0.13 \mathrm{mmol})$, NHC precatalyst 2 (4.2 $\mathrm{mg}, 10.0$ $\mu \mathrm{mol}), \mathrm{Et}_{3} \mathrm{~N}(28 \mu \mathrm{L}, 0.19 \mathrm{mmol})$ and THF $(3 \mathrm{~mL})$ were reacted for $3 \mathrm{~h}$. The crude $(>95: 5 \mathrm{dr})$ was purified by column chromatography (95:5 petrol : EtOAc) to give $\mathbf{1 0}(26 \mathrm{mg}, 69 \%)$ as a colourless oil. $[\alpha]_{\mathrm{D}}^{20}+156\left(c \quad 0.15, \mathrm{CHCl}_{3}\right)$; Chiral HPLC analysis, Chiralpak AD-H (95:5 hexane : IPA, flow rate $\left.1.0 \mathrm{mLmin}^{-1}, 220 \mathrm{~nm}\right) \mathrm{t}_{\mathrm{R}}(R, R) 16.2 \mathrm{~min}, \mathrm{t}_{\mathrm{R}}(S, S) 18.3 \mathrm{~min}, 98: 2 \mathrm{er}$; $v_{\max }\left(\mathrm{ATR}, \mathrm{cm}^{-1}\right) 2954,1773,1734,1661,1454,1437,1323,1259,1045 ;{ }^{1} \mathrm{H}$ NMR (300 MHz, $\left.\mathrm{CDCl}_{3}\right) \delta_{\mathrm{H}}: 0.81\left(3 \mathrm{H}, \mathrm{t}, J 7.1, \mathrm{CH}_{2} \mathrm{CH}_{3}\right), 1.08-1.40\left(5 \mathrm{H}, \mathrm{m}, \mathrm{CH}_{2} \times 2\right.$ and $\left.\mathrm{C}(3) \mathrm{CH}^{\mathrm{A}} \mathrm{H}^{\mathrm{B}}\right), 1.60-$ $1.70\left(1 \mathrm{H}, \mathrm{m}, \mathrm{C}(3) \mathrm{CH}^{\mathrm{A}} H^{\mathrm{B}}\right), 2.79(1 \mathrm{H}, \mathrm{d}, J 6.9, \mathrm{C}(3) H), 3.79(1 \mathrm{H}, \mathrm{t}, J 6.7, \mathrm{C}(4) H), 3.83(3 \mathrm{H}, \mathrm{s}$, $\left.\mathrm{OCH}_{3}\right), 6.68(1 \mathrm{H}, \mathrm{d}, J$ 6.4, C(5)H), $7.06(2 \mathrm{H}, \mathrm{dt}, J 1.8,5.8, \operatorname{ArC}(2) H), 7.23-7.29(3 \mathrm{H}, \mathrm{m}$, $\operatorname{ArC}(3) H$ and $\operatorname{ArC}(4) H) ;{ }^{13} \mathrm{C}\left\{{ }^{1} \mathrm{H}\right\} \operatorname{NMR}\left(100 \mathrm{MHz}, \mathrm{CDCl}_{3}\right) 13.9\left(\mathrm{CH}_{3}\right), 22.5\left(\mathrm{CH}_{2}\right), 26.0\left(\mathrm{CH}_{2}\right)$, $29.4\left(\mathrm{CH}_{2}\right), 41.5(C(4)), 43.4(C(3)), 52.7\left(\mathrm{OCH}_{3}\right), 118.4(C(5)), 128.1(\mathrm{CPhH}-2,6), 128.1$ (CPh-4), 129.1 (ArC(3)), 136.0 (ArC(1)), $142.1(C(6)), 161.1\left(C_{2} \mathrm{Me}\right), 168.8(C(2))$; HRMS $(\mathrm{ESI}+) \mathrm{C}_{17} \mathrm{H}_{24} \mathrm{O}_{4} \mathrm{~N}\left[\mathrm{M}+\mathrm{NH}_{4}\right]^{+}$found 306.1702, requires 306.1700 (+ $\left.0.7 \mathrm{ppm}\right)$. 


\subsection{3 (3S,4S)-Methyl 3-isobutyl-2-oxo-4-phenyl-3,4-dihydro-2H-pyran-6-carboxylate}

11

Following General Procedure 4.3, 4-methyl-1-oxopentan-2-yl 4-nitrobenzoate 7 (133 mg, $0.50 \mathrm{mmol})$, (E)-methyl 2-oxo-4-phenylbut-3-enoate 8 (63.0 $\mathrm{mg}, 0.33 \mathrm{mmol})$, NHC precatalyst $2(12.0 \mathrm{mg}, 33 \mu \mathrm{mol}), \mathrm{Et}_{3} \mathrm{~N}(70 \mu \mathrm{L}, 0.50 \mathrm{mmol})$ and THF $(5 \mathrm{~mL})$ were reacted for $24 \mathrm{~h}$. The crude ( $>95: 5 \mathrm{dr}$ ) was purified by column chromatography (95:5 petrol : EtOAc) to give 11 (60 $\mathrm{mg}, 63 \%)$ as a colourless oil. $[\alpha]_{\mathrm{D}}^{20}+279\left(c\right.$ 0.14, $\left.\mathrm{CHCl}_{3}\right)$; Chiral HPLC analysis, Chiralpak AD-H (95:5 hexane : IPA, flow rate $\left.1.0 \mathrm{mLmin}^{-1}, 220 \mathrm{~nm}\right) \mathrm{t}_{\mathrm{R}}(R, R) 11.9 \mathrm{~min}, \mathrm{t}_{\mathrm{R}}(S, S) 13.0 \mathrm{~min}$, $>99: 1 \mathrm{er} ; v_{\max }\left(\mathrm{KBr}, \mathrm{cm}^{-1}\right) 2959,1769,1708,1661,1317,1265,1094,1082 ;{ }^{1} \mathrm{H}$ NMR (300 $\left.\mathrm{MHz}, \mathrm{CDCl}_{3}\right) \delta_{\mathrm{H}} 0.83\left(3 \mathrm{H}, \mathrm{d}, J\right.$ 6.6, $\left.\mathrm{CH}_{3}\right), 0.88\left(3 \mathrm{H}, \mathrm{d}, J 6.6, \mathrm{CH}_{3}\right), 1.04(1 \mathrm{H}$, app dt, J 7.0, 14.0, $i$ - $\mathrm{BuCH}), 1.56\left(2 \mathrm{H}, \mathrm{dt}, J 7.0,13.9, \mathrm{C}(3) \mathrm{C}^{\mathrm{A}} \mathrm{H}^{\mathrm{B}}\right), 1.72\left(1 \mathrm{H}, \mathrm{dd}, J 7.0,14.0, \mathrm{C}(3) \mathrm{CH}_{\mathrm{a}} \mathrm{H}_{\mathrm{a}}\right)$, $2.93(1 \mathrm{H}$, app q, $J 6.9, \mathrm{C}(3) H), 3.77(1 \mathrm{H}, \mathrm{t}, J 6.7, \mathrm{C}(4) H), 3.87\left(3 \mathrm{H}, \mathrm{s}, \mathrm{OCH}_{3}\right), 6.72(1 \mathrm{H}, \mathrm{d}, J$ 6.4, $\mathrm{C}(5) H), 7.07-7.10(2 \mathrm{H}, \mathrm{dt}, J 1.8,5.8, \operatorname{ArC}(2) H), 7.27-7.34(3 \mathrm{H}, \mathrm{m}, \operatorname{ArC}(3) H$ and $\operatorname{ArC}(4) H) ;{ }^{13} \mathrm{C}\left\{{ }^{1} \mathrm{H}\right\} \mathrm{NMR}\left(100 \mathrm{MHz}, \mathrm{CDCl}_{3}\right) \delta_{\mathrm{C}}: 22.2\left(\left(\mathrm{CH}_{3}\right)_{2} \mathrm{CH}\right), 22.5\left(\left(\mathrm{CH}_{3}\right)_{2} \mathrm{CH}\right), 25.2$ $\left(\left(\mathrm{CH}_{3}\right)_{2} \mathrm{CH}\right), 35.2\left(\mathrm{C}(3) \mathrm{CH}_{2}\right), 41.2(\mathrm{C}(3)), 41.7(\mathrm{C}(4)), 52.7\left(\mathrm{OCH}_{3}\right), 118.4(C(5)), 128.1$ (ArC(2)), 128.1 (ArCC(4)), 129.1 (ArCC(3)), $136.0(\operatorname{ArC}(1)), 142.1(C(6)), 161.1\left(C_{2} \mathrm{Me}\right)$, $168.9(C(2))$; $\mathrm{HRMS}(\mathrm{ESI}+) \mathrm{C}_{17} \mathrm{H}_{24} \mathrm{O}_{4} \mathrm{~N},\left[\mathrm{M}+\mathrm{NH}_{4}\right]^{+}$found 306.1704 ; requires $306.1700(+1.4$ ppm).

\subsection{4 (3S,4S)-Ethyl 3-benzyl-2-oxo-4-(p-tolyl)-3,4-dihydro-2H-pyran-6-carboxylate 12}

Following General Procedure 4.3, 1-oxo-3-phenylpropan-2-yl 4-nitrobenzoate 7 (202 mg, $0.675 \mathrm{mmol})$, (E)-ethyl 4-(p-tolyl)-2-oxobut-3-enoate 36 (98.2 mg, $0.450 \mathrm{mmol}), \mathrm{NHC}$ precatalyst $2(16.6 \mathrm{mg}, 45.0 \mu \mathrm{mol}), \mathrm{Et}_{3} \mathrm{~N}(94.1 \mu \mathrm{L}, 0.675 \mathrm{mmol})$ and $\mathrm{THF}(10 \mathrm{~mL})$ were reacted for $12 \mathrm{~h}$. The crude (94:6 dr) was purified by column chromatography (95:5 petrol : EtOAc) to give 12 (122 mg, 77\%) as a pale yellow oil, with spectroscopic data in accordance with the literature. ${ }^{6 \mathrm{~b}}[\alpha]_{\mathrm{D}}^{20}+299.4\left(c 0.5, \mathrm{CHCl}_{3}\right)\left\{\right.$ Lit. $^{6 \mathrm{~b}}[\alpha]_{\mathrm{D}}^{20}+310.3\left(c\right.$ 1.0, $\left.\left.\mathrm{CHCl}_{3}\right)\right\}$; Chiral HPLC analysis, Chiralcel OD-H (90:10 hexane : IPA, flow rate $\left.0.5 \mathrm{mLmin}^{-1}, 254 \mathrm{~nm}, 30{ }^{\circ} \mathrm{C}\right) \mathrm{t}_{\mathrm{R}}$ $(3 R, 4 R): 19.7 \mathrm{~min}, \mathrm{t}_{\mathrm{R}}(3 S, 4 S): 23.5 \mathrm{~min}, 96: 4 \mathrm{er} ; v_{\max }\left(\right.$ film, $\left.\mathrm{cm}^{-1}\right)$ 2981, 1773, 1732; ${ }^{1} \mathrm{H} \mathrm{NMR}$ $\left(400 \mathrm{MHz}, \mathrm{CDCl}_{3}\right) \delta_{\mathrm{H}}: 1.34\left(3 \mathrm{H}, \mathrm{t}, J 7.1, \mathrm{OCH}_{2} \mathrm{CH}_{3}\right), 2.36\left(3 \mathrm{H}, \mathrm{s}, \mathrm{C}(4) \mathrm{ArC}(4) \mathrm{CH}_{3}\right), 2.43(1 \mathrm{H}$, dd, $J 15.8,10.5, \mathrm{C}(3) H), 3.22-3.32\left(2 \mathrm{H}, \mathrm{m}, \mathrm{C}(3) \mathrm{C} H_{2}\right), 3.60(1 \mathrm{H}, \mathrm{t}, J 6.8, \mathrm{C}(4) H), 4.31$ (2H, q, $J$ 7.1, $\left.\mathrm{OCH}_{2} \mathrm{CH}_{3}\right), 6.66(1 \mathrm{H}, \mathrm{d}, J 6.7, \mathrm{C}(5) H), 6.93\left(2 \mathrm{H}, \mathrm{d}, J 8.1, \mathrm{C}(3) \mathrm{CH}_{2} \mathrm{ArC}(2) H\right), 7.09(2 \mathrm{H}$, $\mathrm{d}, J 6.8, \mathrm{C}(4) \operatorname{ArC}(2) H), 7.16(2 \mathrm{H}, \mathrm{d}, J 7.8, \mathrm{C}(4) \mathrm{ArC}(3) H), 7.21-7.36\left(3 \mathrm{H}, \mathrm{m}, \mathrm{C}(3) \mathrm{CH}_{2} \mathrm{ArC}(3) H\right.$ and $\left.\mathrm{C}(3) \mathrm{CH}_{2} \mathrm{ArC}(4) H\right) ; \quad{ }^{13} \mathrm{C}\left\{{ }^{1} \mathrm{H}\right\} \quad\left(100 \quad \mathrm{MHz}, \quad \mathrm{CDCl}_{3}\right) \quad \delta_{\mathrm{C}}: \quad 14.2 \quad\left(\mathrm{OCH}_{2} \mathrm{CH}_{3}\right), \quad 21.1$ 
(C(4)ArC(4) $\left.\mathrm{CH}_{3}\right), 32.1\left(\mathrm{C}(3) \mathrm{CH}_{2}\right), 40.2(C(4)), 44.9(C(3)), 61.9\left(\mathrm{OCH}_{2} \mathrm{CH}_{3}\right), 118.8(C(5))$, $126.7\left(\mathrm{C}(3) \mathrm{CH}_{2} \mathrm{ArC}(4)\right), \quad 128.3 \quad\left(\mathrm{C}(3) \mathrm{CH}_{2} \mathrm{ArC}(3)\right), \quad 128.6 \quad\left(\mathrm{C}(3) \mathrm{CH}_{2} \mathrm{ArC}(2)\right), \quad 129.0$

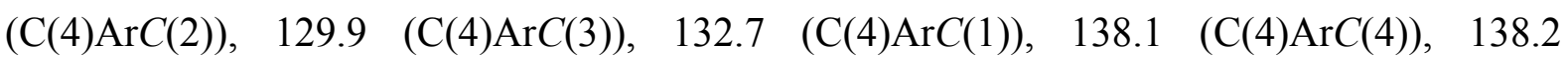
$\left(\mathrm{C}(3) \mathrm{CH}_{2} \mathrm{ArC}(4)\right), 141.9(C(6)), 160.5\left(\mathrm{CO}_{2} \mathrm{Et}\right), 168.7$ (C(2)).

\subsection{5 (3S,4S)-Methyl 3-benzyl-4-(4-methoxyphenyl)-2-oxo-3,4-dihydro-2H-pyran-6- carboxylate 13}

Following General Procedure 4.3, 1-oxo-3-phenylpropan-2-yl 4-nitrobenzoate 7 (202 mg, $0.675 \mathrm{mmol}$ ), (E)-methyl 4-(4-methoxyphenyl)-2-oxobut-3-enoate 37 (99.1 mg, $0.450 \mathrm{mmol}$ ), NHC precatalyst $2(16.6 \mathrm{mg}, 45.0 \mu \mathrm{mol}), \mathrm{Et}_{3} \mathrm{~N}(94.1 \mu \mathrm{L}, 0.675 \mathrm{mmol})$ and THF $(10 \mathrm{~mL})$ were reacted for $16 \mathrm{~h}$. The crude (91:9 dr) was purified by column chromatography (90:10 petrol : EtOAc) to give $13(98.3 \mathrm{mg}, 62 \%)$ as a pale yellow oil. $[\alpha]_{\mathrm{D}}^{20}+277.3\left(c 0.5, \mathrm{CHCl}_{3}\right)$; Chiral HPLC analysis, Chiralcel OD-H (90:10 hexane : IPA, flow rate $\left.0.5 \mathrm{mLmin}^{-1}, 220 \mathrm{~nm}, 30^{\circ} \mathrm{C}\right)$ $\mathrm{t}_{\mathrm{R}}(3 R, 4 R): 40.7 \mathrm{~min}, \mathrm{t}_{\mathrm{R}}(3 S, 4 S): 45.0 \mathrm{~min},>99: 1 \mathrm{er} ; v_{\max }\left(\mathrm{film}, \mathrm{cm}^{-1}\right) 2955,1771,1734 ;{ }^{1} \mathrm{H}$ NMR (300 MHz, $\left.\mathrm{CDCl}_{3}\right) \delta_{\mathrm{H}}: 2.41(1 \mathrm{H}, \mathrm{dd}, J 15.7,10.6, \mathrm{C}(3) H), 3.21-3.30\left(2 \mathrm{H}, \mathrm{m}, \mathrm{C}(3) \mathrm{CH}_{2}\right)$, $3.58(1 \mathrm{H}, \mathrm{t}, J 6.7, \mathrm{C}(4) H), 3.80\left(3 \mathrm{H}, \mathrm{s}, \mathrm{OCH}_{3}\right), 3.84\left(3 \mathrm{H}, \mathrm{s}, \mathrm{CO}_{2} \mathrm{CH}_{3}\right), 6.65(1 \mathrm{H}, \mathrm{d}, J 6.8, \mathrm{C}(5) H)$, $6.86(2 \mathrm{H}, \mathrm{d}, J$ 8.8, C(4)ArC(2)H), $6.93(2 \mathrm{H}, \mathrm{d}, J$ 8.8, C(4)ArC(3)H), 7.04-7.11 (2H, m, $\left.\mathrm{C}(3) \mathrm{CH}_{2} \mathrm{ArC}(2) H\right), 7.20-7.35\left(3 \mathrm{H}, \mathrm{m}, \mathrm{C}(3) \mathrm{CH}_{2} \mathrm{ArC}(3) H\right.$ and $\left.\mathrm{C}(3) \mathrm{CH}_{2} \mathrm{ArC}(4) H\right) ;{ }^{13} \mathrm{C}\left\{{ }^{1} \mathrm{H}\right\}$ NMR (100 MHz, $\left.\mathrm{CDCl}_{3}\right) \delta_{\mathrm{C}}: 30.9\left(\mathrm{C}(3) \mathrm{CH}_{2}\right), 38.7(\mathrm{C}(4)), 43.9(\mathrm{C}(3)), 51.6\left(\mathrm{CO}_{2} \mathrm{CH}_{3}\right), 54.3$ (C(4)ArC(4)OCH$), 113.5$ (C(4)ArC(2)), 118.1 (C(5)), 125.7 (C(3) $\left.\mathrm{CH}_{2} \mathrm{ArC}(1)\right), 126.4$ (C(4)ArC(1)), 127.6 (C(3) $\left.\mathrm{CH}_{2} \mathrm{ArC}(3)\right), 127.9$ (C(3) $\left.\mathrm{CH}_{2} \mathrm{ArC}(2)\right), 128.9$ (C(4)ArC(3)), 137.1 $\left(\mathrm{C}(3) \mathrm{CH}_{2} \mathrm{ArC}(1)\right), 140.5(C(6)), 158.5(\mathrm{C}(4) \mathrm{ArC}(4)), 160.0\left(\mathrm{CO}_{2} \mathrm{Me}\right), 167.6(C(2))$; HRMS $\left(\mathrm{NSI}^{+}\right) \mathrm{C}_{21} \mathrm{H}_{24} \mathrm{O}_{5} \mathrm{~N}_{1}\left[\mathrm{M}+\mathrm{NH}_{4}\right]^{+}$found 370.1655, requires 370.1649 (+1.6 ppm).

\subsection{6 (3S,4S)-Ethyl 3-butyl-2-oxo-4-(p-tolyl)-3,4-dihydro-2H-pyran-6-carboxylate 14}

Following General Procedure 4.3, 1-oxohexan-2-yl 4-nitrobenzoate (200 mg, $0.750 \mathrm{mmol})$, (E)-ethyl 2-oxo-4-(p-tolyl)but-3-enoate 36 (109 mg, $0.500 \mathrm{mmol})$, NHC precatalyst 2 (18.4 $\mathrm{mg}, 50.0 \mu \mathrm{mol}), \mathrm{Et}_{3} \mathrm{~N}(105 \mu \mathrm{L}, 0.750 \mathrm{mmol})$ and THF $(10 \mathrm{~mL})$ were reacted for $9 \mathrm{~h}$. The crude (92:8 dr) was purified by column chromatography (95:5 petrol : EtOAc) to give 14 (110 mg, $70 \%)$ as a pale yellow oil. $[\alpha]_{\mathrm{D}}^{20}+249.2\left(c 0.5, \mathrm{CHCl}_{3}\right)$; Chiral HPLC analysis, Chiralpak AD$\mathrm{H}\left(95: 5\right.$ hexane : IPA, flow rate $\left.1 \mathrm{mLmin}^{-1}, 254 \mathrm{~nm}, 30^{\circ} \mathrm{C}\right) \mathrm{t}_{\mathrm{R}}(3 S, 4 S): 11.3 \mathrm{~min}, \mathrm{t}_{\mathrm{R}}(3 R, 4 R)$ : 13.7 min, >99: $1 \mathrm{er} ; v_{\max }\left(\right.$ film, $\left.\mathrm{cm}^{-1}\right)$ 2957, 1773, 1732; ${ }^{1} \mathrm{H}$ NMR (400 MHz, $\left.\mathrm{CDCl}_{3}\right) \delta_{\mathrm{H}}: 0.78$ $\left(3 \mathrm{H}, \mathrm{t}, J\right.$ 7.2, $\left.\mathrm{CH}_{2} \mathrm{CH}_{3}\right), 1.04-1.38\left(7 \mathrm{H}, \mathrm{m},\left(\mathrm{CH}_{2}\right)_{2}\right.$ and $\left.\mathrm{OCH}_{2} \mathrm{CH}_{3}\right), 1.54-1.71\left(2 \mathrm{H}, \mathrm{m}, \mathrm{CH}_{2}\right)$, $2.24\left(3 \mathrm{H}, \mathrm{s}, \operatorname{ArC}(4) \mathrm{CH}_{3}\right), 2.73(1 \mathrm{H}, \mathrm{q}, J 7.0, \mathrm{C}(3) H), 3.70(1 \mathrm{H}, \mathrm{t}, J 6.8, \mathrm{C}(4) H), 4.24(2 \mathrm{H}, \mathrm{q}, J$ 
7.1, $\left.\mathrm{OCH}_{2} \mathrm{CH}_{3}\right), 6.62(1 \mathrm{H}, \mathrm{d}, J 6.4, \mathrm{C}(5) H), 6.91(2 \mathrm{H}, \mathrm{d}, J 8.1, \operatorname{ArC}(2) H), 7.04(2 \mathrm{H}, \mathrm{d}, J 7.8$, $\operatorname{ArC}(3) H) ;{ }^{13} \mathrm{C}\left\{{ }^{1} \mathrm{H}\right\}$ NMR $\left(100 \mathrm{MHz}, \mathrm{CDCl}_{3}\right) \delta_{\mathrm{C}}: 13.9\left(\mathrm{OCH}_{2} \mathrm{CH}_{3}\right), 14.2\left(\mathrm{CH}_{2} \mathrm{CH}_{3}\right), 21.1$ $\left(\mathrm{ArC}(4) \mathrm{CH}_{3}\right), 22.5\left(\mathrm{CH}_{2}\right), 26.0\left(\mathrm{CH}_{2}\right), 29.4\left(\mathrm{CH}_{2}\right), 41.1(\mathrm{C}(4)), 43.4(\mathrm{C}(3)), 61.9\left(\mathrm{OCH}_{2} \mathrm{CH}_{3}\right)$, $118.4(C(5)), 127.9(\operatorname{ArC}(2)), 129.8(\operatorname{ArC}(3)), 132.9(\operatorname{ArC}(1)), 137.9(\operatorname{ArC}(4)), 142.1(C(6))$, $160.6\left(\mathrm{CO}_{2} \mathrm{Et}\right), 169.0(C(2))$; $\mathrm{HRMS}\left(\mathrm{NSI}^{+}\right) \mathrm{C}_{19} \mathrm{H}_{28} \mathrm{O}_{4} \mathrm{~N}\left[\mathrm{M}+\mathrm{NH}_{4}\right]^{+}$found 334.2019, requires $334.2013(+1.8 \mathrm{ppm})$.

\subsection{7 (3S,4S)-Methyl 3-butyl-4-(4-methoxyphenyl)-2-oxo-3,4-dihydro-2H-pyran-6- carboxylate 15}

Following General Procedure 4.3, 1-oxohexan-2-yl 4-nitrobenzoate (200 mg, $0.750 \mathrm{mmol}$ ), (E)-methyl 4-(4-methoxyphenyl)-2-oxobut-3-enoate 37 (110 mg, $0.500 \mathrm{mmol})$, NHC precatalyst $2(18.4 \mathrm{mg}, 50.0 \mu \mathrm{mol}), \mathrm{Et}_{3} \mathrm{~N}(105 \mu \mathrm{L}, 0.750 \mathrm{mmol})$ and THF $(10 \mathrm{~mL})$ were reacted for $14 \mathrm{~h}$. The crude (92:8 dr) was purified by column chromatography (95:5 petrol : EtOAc) to give $15(114 \mathrm{mg}, 72 \%)$ as a pale yellow oil. $[\alpha]_{\mathrm{D}}^{20}+245.1\left(c 0.5, \mathrm{CHCl}_{3}\right)$; Chiral HPLC analysis, Chiralpak AD-H (90:10 hexane : IPA, flow rate $\left.0.5 \mathrm{mLmin}^{-1}, 220 \mathrm{~nm}, 30{ }^{\circ} \mathrm{C}\right) \mathrm{t}_{\mathrm{R}}(3 S, 4 S): 30.5$ $\min , \mathrm{t}_{\mathrm{R}}(3 R, 4 R): 32.6 \mathrm{~min},>99: 1 \mathrm{er} ; v_{\max }\left(\mathrm{film}, \mathrm{cm}^{-1}\right)$ 2955, 1771, 1734; ${ }^{1} \mathrm{H}$ NMR $(300 \mathrm{MHz}$, $\left.\mathrm{CDCl}_{3}\right) \delta_{\mathrm{H}}: 0.84\left(3 \mathrm{H}, \mathrm{t}, J 7.1, \mathrm{CH}_{2} \mathrm{CH}_{3}\right), 1.09-1.43\left(5 \mathrm{H}, \mathrm{m},\left(\mathrm{CH}_{2}\right)_{2}\right.$ and $\left.\mathrm{C}(3) \mathrm{CH}^{\mathrm{A}} \mathrm{H}^{\mathrm{B}}\right), 1.58-$ $1.76\left(1 \mathrm{H}, \mathrm{m}, \mathrm{C}(3) \mathrm{CH}^{\mathrm{A}} H^{\mathrm{B}}\right), 2.78(1 \mathrm{H}, \mathrm{q}, J 6.9, \mathrm{C}(3) H), 3.73-3.80\left(4 \mathrm{H}, \mathrm{m}, \mathrm{ArC}(4) \mathrm{OCH}_{3}\right.$ and $\mathrm{C}(4) H), 3.85\left(3 \mathrm{H}, \mathrm{s}, \mathrm{CO}_{2} \mathrm{CH}_{3}\right), 6.69(1 \mathrm{H}, \mathrm{d}, J 6.5, \mathrm{C}(5) H), 6.83(2 \mathrm{H}, \mathrm{d}, J 8.7, \operatorname{ArC}(2) H), 7.00$ $(2 \mathrm{H}, \mathrm{d}, J$ 8.7, $\operatorname{ArC}(3) H) ;{ }^{13} \mathrm{C}\left\{{ }^{1} \mathrm{H}\right\} \mathrm{NMR}\left(75 \mathrm{MHz}, \mathrm{CDCl}_{3}\right) \delta_{\mathrm{C}}: 12.8\left(\mathrm{CH}_{2} \mathrm{CH}_{3}\right), 21.4\left(\mathrm{CH}_{2}\right), 24.9$ $\left(\mathrm{CH}_{2}\right), 28.3\left(\mathrm{CH}_{2}\right), 39.6(\mathrm{C}(4)), 42.5(\mathrm{C}(3)), 51.6\left(\mathrm{CO}_{2} \mathrm{CH}_{3}\right), 54.3\left(\mathrm{ArC}(4) \mathrm{OCH}_{3}\right), 113.4$ (ArC(2)), 117.7 (C(5)), 126.7 (ArC(1)), 128.1 (ArC(3)), 140.8 (C(6)), 158.3 (ArC(4)), 160.1 $\left(\mathrm{CO}_{2} \mathrm{Me}\right), 167.9(C(2))$; HRMS $\left(\mathrm{NSI}^{+}\right) \mathrm{C}_{18} \mathrm{H}_{26} \mathrm{O}_{5} \mathrm{~N}\left[\mathrm{M}+\mathrm{NH}_{4}\right]^{+}$found 336.1812, requires 336.1805 (+1.9 ppm).

\subsection{8 (3S,4S)-Methyl}

4-(4-bromophenyl)-3-butyl-2-oxo-3,4-dihydro-2H-pyran-6carboxylate 16

Following General Procedure 4.3, 1-oxohexan-2-yl-4-nitrobenzoate (200 mg, $0.750 \mathrm{mmol})$, (E)-methyl 4-(4-bromophenyl)-2-oxobut-3-enoate 39 (135 mg, $0.500 \mathrm{mmol}$ ), NHC precatalyst $2(18.4 \mathrm{mg}, 50.0 \mu \mathrm{mol}), \mathrm{Et}_{3} \mathrm{~N}(105 \mu \mathrm{L}, 0.750 \mathrm{mmol})$ and THF $(10 \mathrm{~mL})$ were for $4 \mathrm{~h}$. The crude (89:11 dr) was purified by column chromatography (95:5 petrol : EtOAc) to give $\mathbf{1 6}(77.1 \mathrm{mg}$, $42 \%)$ as a pale yellow oil. $[\alpha]_{\mathrm{D}}^{20}+205.3\left(c 0.5, \mathrm{CHCl}_{3}\right)$; Chiral HPLC analysis, Chiralpak AD$\mathrm{H}\left(95: 5\right.$ hexane : IPA, flow rate $\left.1 \mathrm{mLmin}^{-1}, 220 \mathrm{~nm}, 30^{\circ} \mathrm{C}\right) \mathrm{t}_{\mathrm{R}}(3 S, 4 S): 15.1 \mathrm{~min}, \mathrm{t}_{\mathrm{R}}(3 R, 4 R)$ : $17.7 \mathrm{~min}, 99: 1 \mathrm{er} ; v_{\max }\left(\right.$ film, $\left.\mathrm{cm}^{-1}\right) 2955,1773,1732 ;{ }^{1} \mathrm{H} \mathrm{NMR}\left(300 \mathrm{MHz}, \mathrm{CDCl}_{3}\right) \delta_{\mathrm{H}}: 0.78-$ 
$1.00\left(3 \mathrm{H}, \mathrm{m}, \mathrm{CH}_{2} \mathrm{CH}_{3}\right), 1.06-1.47\left(5 \mathrm{H}, \mathrm{m},\left(\mathrm{CH}_{2}\right)_{2}\right.$ and $\left.\mathrm{C}(3) \mathrm{CH}^{\mathrm{A}} \mathrm{H}^{\mathrm{B}}\right), 1.54-1.77(1 \mathrm{H}, \mathrm{m}$, $\left.\mathrm{C}(3) \mathrm{CH}^{\mathrm{A}} H^{\mathrm{B}}\right), 2.82(1 \mathrm{H}, \mathrm{q}, J 7.0, \mathrm{C}(3) H), 3.79(1 \mathrm{H}, \mathrm{t}, J 6.7, \mathrm{C}(4) H), 3.87\left(3 \mathrm{H}, \mathrm{s}, \operatorname{ArC}(4) \mathrm{OCH}_{3}\right)$, $6.67(1 \mathrm{H}, \mathrm{d}, J 6.4, \mathrm{C}(5) H), 6.97(2 \mathrm{H}, \mathrm{d}, J 8.4, \operatorname{ArC}(3) H), 7.44(2 \mathrm{H}, \mathrm{d}, J 8.5, \operatorname{ArC}(2) H) ;{ }^{13} \mathrm{C}\left\{{ }^{1} \mathrm{H}\right\}$ $\operatorname{NMR}\left(75 \mathrm{MHz}, \mathrm{CDCl}_{3}\right) \delta_{\mathrm{C}}: 12.8\left(\mathrm{CH}_{2} \mathrm{CH}_{3}\right), 21.4\left(\mathrm{CH}_{2}\right), 24.9\left(\mathrm{CH}_{2}\right), 28.3\left(\mathrm{CH}_{2}\right), 39.7(C(4))$, $42.2(C(3)), 51.7\left(\mathrm{CO}_{2} \mathrm{CH}_{3}\right), 116.6(C(5)), 121.1$ (ArC(4)), 128.7 (ArC(3)), $131.3(\mathrm{ArC}(2))$, 134.0 (ArC(1)), $141.3(C(6)), 159.8\left(C_{2} \mathrm{Me}\right), 167.4(C(2))$; HRMS $\left(\mathrm{NSI}^{+}\right) \mathrm{C}_{17} \mathrm{H}_{23} \mathrm{BrO}_{5} \mathrm{~N}$ $\left[\mathrm{M}+\mathrm{NH}_{4}\right]^{+}$found 384.0813 , requires $384.0805(+2.1 \mathrm{ppm})$.

\subsection{9 (3S,4S)-Methyl 3-butyl-4-(naphthalen-2-yl)-2-oxo-3,4-dihydro-2H-pyran-6- carboxylate 17}

Following General Procedure 4.3, 1-oxohexan-2-yl 4-nitrobenzoate (200 mg, $0.750 \mathrm{mmol})$, (E)-methyl 4-(naphthalen-2-yl)-2-oxobut-3-enoate 40 (120 mg, $0.500 \mathrm{mmol})$, NHC precatalyst $2(18.4 \mathrm{mg}, 50.0 \mu \mathrm{mol}), \mathrm{Et}_{3} \mathrm{~N}(105 \mu \mathrm{L}, 0.750 \mathrm{mmol})$ and THF $(10 \mathrm{~mL})$ were reacted for $6 \mathrm{~h}$. The crude (94:6 dr) was purified by column chromatography (95:5 petrol : EtOAc) to give 17 $(81.7 \mathrm{mg}, 48 \%)$ as a colourless solid. mp $99-101^{\circ} \mathrm{C} ;[\alpha]_{\mathrm{D}}^{20}+297.0\left(c 0.5, \mathrm{CHCl}_{3}\right)$; Chiral HPLC analysis, Chiralpak AD-H (95:5 hexane : IPA, flow rate $\left.1 \mathrm{mLmin}^{-1}, 254 \mathrm{~nm}, 30^{\circ} \mathrm{C}\right) \mathrm{t}_{\mathrm{R}}(3 R, 4 R)$ : $22.0 \mathrm{~min}, \mathrm{t}_{\mathrm{R}}(3 S, 4 S): 25.0 \mathrm{~min},>99: 1 \mathrm{er} ; v_{\max }$ (solid) 2951, 1757, 1736; ${ }^{1} \mathrm{H}$ NMR $(400 \mathrm{MHz}$, $\left.\mathrm{CDCl}_{3}\right) \delta_{\mathrm{H}}: 0.83\left(3 \mathrm{H}, \mathrm{t}, J 7.3, \mathrm{CH}_{2} \mathrm{CH}_{3}\right), 1.11-1.49\left(5 \mathrm{H}, \mathrm{m},\left(\mathrm{CH}_{2}\right)_{2}\right.$ and $\left.\mathrm{C}(3) \mathrm{CH}^{\mathrm{A}} \mathrm{H}^{\mathrm{B}}\right), 1.63-$ $1.78\left(1 \mathrm{H}, \mathrm{m}, \mathrm{C}(3) \mathrm{CH}^{\mathrm{A}} H^{\mathrm{B}}\right), 2.90(1 \mathrm{H}, \mathrm{q}, J 7.0, \mathrm{C}(3) H), 3.88\left(3 \mathrm{H}, \mathrm{s}, \mathrm{CO}_{2} \mathrm{CH}_{3}\right), 3.99(1 \mathrm{H}, \mathrm{t}, J 6.7$, $\mathrm{C}(4) H), 6.77(1 \mathrm{H}, \mathrm{d}, J 6.3, \mathrm{C}(5) H), 7.19(1 \mathrm{H}, \mathrm{dd}, J$ 8.5, 1.9, $\operatorname{Ar} H), 7.44-7.52$ (2H, m, ArH), $7.57(1 \mathrm{H}, \mathrm{d}, J 1.8, \operatorname{ArC}(2) H), 7.74-7.85(3 \mathrm{H}, \mathrm{m}, \operatorname{Ar} H) ;{ }^{13} \mathrm{C}\left\{{ }^{1} \mathrm{H}\right\} \mathrm{NMR}\left(75 \mathrm{MHz}, \mathrm{CDCl}_{3}\right) \delta_{\mathrm{C}}$ : $12.8\left(\mathrm{CH}_{3} \mathrm{CH}_{2}\right), 21.4\left(\mathrm{CH}_{2}\right), 25.0\left(\mathrm{CH}_{2}\right), 28.4\left(\mathrm{CH}_{2}\right), 40.6(\mathrm{C}(4)), 42.4(\mathrm{C}(3)), 51.7\left(\mathrm{CO}_{2} \mathrm{CH}_{3}\right)$, $117.2(C(5)), 124.5(\operatorname{ArC}(8)), 125.3(\operatorname{ArC}), 125.5(\operatorname{ArC}), 126.1(\operatorname{ArC}(2)), 126.6(\operatorname{ArC}(6)), 126.8$ (ArC), 127.7 (ArC(2a)), $128.0(\operatorname{ArC}), 131.9$ (ArC(1)), 132.4 (ArC(6a)), 141.1 (C(5)), 163.3 $\left(\mathrm{CO}_{2} \mathrm{CH}_{3}\right), 167.7(C(2))$; HRMS $\left(\mathrm{NSI}^{+}\right) \mathrm{C}_{21} \mathrm{H}_{26} \mathrm{O}_{4} \mathrm{~N}_{1}\left[\mathrm{M}+\mathrm{NH}_{4}\right]^{+}$found 356.1862, requires $356.1856(+1.6 \mathrm{ppm})$.

\subsection{General procedure for the synthesis of $\alpha, \beta$-unsaturated $\boldsymbol{\gamma}$-ketoesters}

Based upon a literature procedure, ${ }^{21}$ periodic acid (1 eq.) was added portionwise to a solution of $(+)$-diethyl L-tartrate (1 eq.) in anhydrous $\mathrm{Et}_{2} \mathrm{O}(0.5 \mathrm{M})$. The solution was stirred at $\mathrm{rt}$ for $3 \mathrm{~h}$ before being filtered into a dried two-necked round-bottomed flask containing $\mathrm{MgSO}_{4}(1.0 \mathrm{~g} /$ $7 \mathrm{~mL} \mathrm{Et}_{2} \mathrm{O}$ ), washing with THF ( $1 \mathrm{~mL} / 0.8 \mathrm{~mL}$ EtO). The resulting solution was cooled to 0 ${ }^{\circ} \mathrm{C}$ before the appropriate phosphorane (1.5 eq.) was added in one portion. The reaction was stirred overnight ( $c f .16 \mathrm{~h}$ ), allowing to warm slowly to rt. The reaction was filtered and 
concentrated under reduced pressure to give the crude product, which was purified by column chromatography.

\subsection{1 (E)-Ethyl 4-oxopent-2-enoate 41}

Following General Procedure 4.4, periodic acid (0.95 g, $4.2 \mathrm{mmol})$ and (+)-diethyl L-tartrate $(0.72 \mathrm{~mL}, 4.2 \mathrm{mmol})$ in $\mathrm{Et}_{2} \mathrm{O}(8.5 \mathrm{~mL})$ were stirred for $3 \mathrm{~h}$ at $\mathrm{rt}$. The reaction was filtered into a two-necked round-bottomed flask containing $\mathrm{MgSO}_{4}(1.0 \mathrm{~g})$, washing with THF $(10.5 \mathrm{~mL})$, before 1-(triphenylphosphoranylidene)propan-2-one $(2.0 \mathrm{~g}, 6.3 \mathrm{mmol})$ was added at $0{ }^{\circ} \mathrm{C}$. The crude product was purified by column chromatography (80:20 hexane : $\left.\mathrm{Et}_{2} \mathrm{O}, \mathrm{R}_{f} 0.24\right)$ to give $41(0.61 \mathrm{~g}, 68 \%)$ as a colourless oil, with spectroscopic data in accordance with the literature. ${ }^{22}$ ${ }^{1} \mathrm{H}$ NMR $\left(300 \mathrm{MHz}, \mathrm{CDCl}_{3}\right) \delta_{\mathrm{H}}: 1.32\left(3 \mathrm{H}, \mathrm{t}, J 7.2 \mathrm{CH}_{2} \mathrm{CH}_{3}\right), 2.36\left(3 \mathrm{H}, \mathrm{s}, \mathrm{COCH}_{3}\right), 4.26(2 \mathrm{H}$, q, $\left.J 7.2, \mathrm{OCH}_{2}\right), 6.64\left(1 \mathrm{H}, \mathrm{d}, J 16.1, \mathrm{EtO}_{2} \mathrm{CCH}\right), 7.01(1 \mathrm{H}, \mathrm{d}, J 16.1, \mathrm{CHCOMe})$.

\subsection{2 (E)-Ethyl 4-oxo-4-(p-tolyl)but-2-enoate 42}

Following General Procedure 4.4, periodic acid $(0.39 \mathrm{~g}, 1.7 \mathrm{mmol})$ and $(+)$-diethyl L-tartrate $(0.35 \mathrm{~g}, 1.7 \mathrm{mmol})$ in $\mathrm{Et}_{2} \mathrm{O}(3.5 \mathrm{~mL})$ were stirred for $3 \mathrm{~h}$ at $\mathrm{rt}$. The reaction was filtered into a two-necked round-bottomed flask containing $\mathrm{MgSO}_{4}(0.5 \mathrm{~g})$, washing with THF $(4.3 \mathrm{~mL})$, before 1-(p-tolyl)-2-(triphenylphosphoranylidene)ethanone $(1.0 \mathrm{~g}, 2.5 \mathrm{mmol})$ was added at 0 ${ }^{\circ} \mathrm{C}$. The crude product was purified by column chromatography (95:5 petrol : EtOAc, $\left.\mathrm{R}_{f} 0.36\right)$ to give $42(0.39 \mathrm{~g}, 78 \%)$ as a yellow oil, with spectroscopic data in accordance with the literature. ${ }^{21}{ }^{1} \mathrm{H}$ NMR $\left(300 \mathrm{MHz}, \mathrm{CDCl}_{3}\right) \delta_{\mathrm{H}}: 1.35\left(3 \mathrm{H}, \mathrm{t}, J 7.1, \mathrm{CH}_{2} \mathrm{CH}_{3}\right), 2.44(3 \mathrm{H}, \mathrm{s}$, $\left.\mathrm{ArC}(4) \mathrm{CH}_{3}\right), 4.30\left(2 \mathrm{H}, \mathrm{q}, J\right.$ 7.1, $\left.\mathrm{OCH}_{2}\right), 6.87\left(1 \mathrm{H}, \mathrm{d}, J 15.6, \mathrm{EtO}_{2} \mathrm{CCH}\right), 7.31(2 \mathrm{H}, \mathrm{d}, J 8.2$, $\operatorname{ArC}(3,5) H), 7.91(1 \mathrm{H}, \mathrm{d}, J 15.5, \mathrm{CHCOAr}), 7.91(1 \mathrm{H}, \mathrm{d}, J 8.2, \operatorname{ArC}(2,6) H)$.

\subsection{3 (E)-Ethyl 4-(4-methoxyphenyl)-4-oxobut-2-enoate 43}

Following General Procedure 4.4, periodic acid $(0.37 \mathrm{~g}, 1.6 \mathrm{mmol})$ and $(+)$-diethyl L-tartrate $(0.34 \mathrm{~g}, 1.6 \mathrm{mmol})$ in $\mathrm{Et}_{2} \mathrm{O}(3.2 \mathrm{~mL})$ were stirred for $3 \mathrm{~h}$ at $\mathrm{rt}$. The reaction was filtered into a two-necked round-bottomed flask containing $\mathrm{MgSO}_{4}(0.5 \mathrm{~g})$, washing with THF $(4.1 \mathrm{~mL})$, before 1-(4-methoxyphenyl)-2-(triphenylphosphoranylidene)ethanone (1.0 g, $2.4 \mathrm{mmol})$ was added at $0{ }^{\circ} \mathrm{C}$. The crude product was purified by column chromatography (70:30 petrol : $\mathrm{Et}_{2} \mathrm{O}$, $\left.\mathrm{R}_{f} 0.25\right)$ to give $43(0.48 \mathrm{~g}, 83 \%)$ as a yellow oil, which solidified over time, with spectroscopic data in accordance with the literature. ${ }^{21} \mathrm{mp} 40-42{ }^{\circ} \mathrm{C} ;{ }^{1} \mathrm{H}$ NMR $\left(300 \mathrm{MHz}, \mathrm{CDCl}_{3}\right) \delta_{\mathrm{H}}: 1.35$ $\left(3 \mathrm{H}, \mathrm{t}, J 7.2 \mathrm{CH}_{2} \mathrm{CH}_{3}\right), 3.90\left(3 \mathrm{H}, \mathrm{s}, \mathrm{OCH}_{3}\right), 4.30\left(2 \mathrm{H}, \mathrm{q}, J 7.1, \mathrm{OCH}_{2}\right), 6.87(1 \mathrm{H}, \mathrm{d}, J 15.5$, $\left.\mathrm{EtO}_{2} \mathrm{CCH}\right), 6.98(2 \mathrm{H}, \mathrm{d}, J$ 9.0, $\operatorname{ArC}(3,5) H), 7.92(1 \mathrm{H}, \mathrm{d}, J 15.5, \mathrm{CHCOAr}), 8.01(2 \mathrm{H}, \mathrm{d}, J 9.0$, $\operatorname{ArC}(2,6) H)$. 


\subsection{4 (E)-Ethyl 4-(4-chlorophenyl)-4-oxobut-2-enoate 44}

Following General Procedure 4.4, periodic acid (0.37 g, $1.6 \mathrm{mmol})$ and (+)-diethyl L-tartrate $(0.33 \mathrm{~g}, 1.6 \mathrm{mmol})$ in $\mathrm{Et}_{2} \mathrm{O}(3.2 \mathrm{~mL})$ were stirred for $3 \mathrm{~h}$ at $\mathrm{rt}$. The reaction was filtered into a two-necked round-bottomed flask containing $\mathrm{MgSO}_{4}(0.5 \mathrm{~g})$, washing with THF (4.0 mL), before 1-(4-chlorophenyl)-2-(triphenylphosphoranylidene)ethanone (1.0 g, $2.4 \mathrm{mmol})$ was added at $0{ }^{\circ} \mathrm{C}$. The crude product was purified by column chromatography (95:5 petrol : EtOAc, $\left.\mathrm{R}_{f} 0.29\right)$ to give $44(0.57 \mathrm{~g}, 99 \%)$ as a yellow solid, with spectroscopic data in accordance with the literature. ${ }^{21} \mathrm{mp} 62-63{ }^{\circ} \mathrm{C} ;{ }^{1} \mathrm{H}$ NMR $\left(300 \mathrm{MHz}, \mathrm{CDCl}_{3}\right) \delta_{\mathrm{H}}: 1.35\left(3 \mathrm{H}, \mathrm{t}, J 7.1, \mathrm{CH}_{3}\right), 4.30$ $\left(2 \mathrm{H}, \mathrm{q}, J 7.1, \mathrm{OCH}_{2}\right), 6.90\left(1 \mathrm{H}, \mathrm{d}, J 15.5, \mathrm{EtO}_{2} \mathrm{CCH}\right), 7.49(2 \mathrm{H}, \mathrm{d}, J 8.7, \operatorname{ArC}(3,5) H), 7.87(1 \mathrm{H}$, d, $J$ 15.6, CHCOAr), $7.95(1 \mathrm{H}, \mathrm{d}, J 8.7, \operatorname{ArC}(2,6) H)$.

\subsection{5 (E)-Ethyl 4-oxo-4-(4-(trifluoromethyl)phenyl)but-2-enoate 45}

Following General Procedure 4.4, periodic acid (0.68 g, $3.0 \mathrm{mmol})$ and (+)-diethyl L-tartrate $(0.51 \mathrm{~mL}, 3.0 \mathrm{mmol})$ in $\mathrm{Et}_{2} \mathrm{O}(6.0 \mathrm{~mL})$ were stirred for $3 \mathrm{~h}$ at $\mathrm{rt}$. The reaction was filtered into a two-necked round-bottomed flask containing $\mathrm{MgSO}_{4}(1.0 \mathrm{~g})$, washing with THF $(7.5 \mathrm{~mL})$, before 1-(4-(trifluoromethyl)phenyl)-2-(triphenylphosphoranylidene)ethanone $(2.0 \mathrm{~g}, 4.5$ mmol) was added at $0{ }^{\circ} \mathrm{C}$. The crude product was purified by column chromatography (90:10 petrol : $\left.\mathrm{Et}_{2} \mathrm{O}, \mathrm{R}_{f} 0.24\right)$ to $45\left(0.85 \mathrm{~g}, 70 \%\right.$ ) as a yellow solid. $\mathrm{mp} 61-63{ }^{\circ} \mathrm{C} ; v_{\max }\left(\mathrm{film}, \mathrm{cm}^{-1}\right)$ 1721, 1672, 1630, 1414, 1315, 1294, 1163, 1123, 1111; ${ }^{1} \mathrm{H}$ NMR $\left(300 \mathrm{MHz}, \mathrm{CDCl}_{3}\right) \delta_{\mathrm{H}}: 1.35$ $\left(3 \mathrm{H}, \mathrm{t}, J 7.1 \mathrm{CH}_{3}\right), 4.31$ (2H. q. $\left.J 7.1, \mathrm{OCH}_{2}\right), 6.91\left(1 \mathrm{H}, \mathrm{d}, J 15.6, \mathrm{EtO}_{2} \mathrm{CCH}\right), 7.78(2 \mathrm{H}, \mathrm{d}, J$ 8.4, $\operatorname{ArC}(3,5) H), 7.87(1 \mathrm{H}, \mathrm{d}, J 15.5, \mathrm{CHCOAr}), 8.09(2 \mathrm{H}, \mathrm{d}, J 8.3, \operatorname{ArC}(2,6) H) ;{ }^{13} \mathrm{C}\left\{{ }^{1} \mathrm{H}\right\} \mathrm{NMR}$ $\left(75 \mathrm{MHz}, \mathrm{CDCl}_{3}\right) \delta_{\mathrm{C}}: 14.3\left(\mathrm{CH}_{3}\right), 61.7\left(\mathrm{OCH}_{2}\right), 123.6\left(\mathrm{q},{ }^{1} J_{\mathrm{CF}} 272.8, C \mathrm{~F}_{3}\right), 126.1\left(\mathrm{q},{ }^{3} J_{\mathrm{CF}} 3.7\right.$, $\operatorname{ArC}(2) \mathrm{H}), 129.3(\operatorname{ArC}(3) \mathrm{H}), 133.8\left(\mathrm{EtO}_{2} \mathrm{C}=\mathrm{CH}\right), 135.1 \quad\left(\mathrm{q},{ }^{2} J_{\mathrm{CF}} 32.8, \operatorname{ArC}(1)\right), 135.7$ $\left(\mathrm{C}=\right.$ CHCOAr), $139.4(\mathrm{ArC}(4)), 165.4\left(\mathrm{CO}_{2} \mathrm{Et}\right), 188.9(\mathrm{COAr}) ;{ }^{19} \mathrm{~F}\left\{{ }^{1} \mathrm{H}\right\} \mathrm{NMR}(282 \mathrm{MHz}$, $\left.\mathrm{CDCl}_{3}\right) \delta_{\mathrm{F}}:-63.69\left(\mathrm{CF}_{3}\right)$; HRMS $\left(\mathrm{APCI}^{+}\right) \mathrm{C}_{13} \mathrm{H}_{12} \mathrm{O}_{3} \mathrm{~F}_{3}[\mathrm{M}+\mathrm{H}]^{+}$found 273.0732, requires $273.0733(-0.4 \mathrm{ppm})$.

\subsection{6 (E)-Ethyl 4-(naphthalen-2-yl)-4-oxobut-2-enoate 46}

Following General Procedure 4.4, periodic acid (1.06 g, $4.6 \mathrm{mmol})$ and (+)-diethyl L-tartrate $(0.80 \mathrm{~mL}, 4.6 \mathrm{mmol})$ in $\mathrm{Et}_{2} \mathrm{O}(9.0 \mathrm{~mL})$ were stirred for $3 \mathrm{~h}$ at $\mathrm{rt}$. The reaction was filtered into a two-necked round-bottomed flask containing $\mathrm{MgSO}_{4}(1.5 \mathrm{~g})$, washing with THF (11.5 mL), before 1-(naphthalen-2-yl)-2-(triphenylphosphoranylidene)ethanone (3.00 g, $7.0 \mathrm{mmol})$ was added at $0{ }^{\circ} \mathrm{C}$. The crude product was purified by column chromatography (80:20 hexane : $\left.\mathrm{Et}_{2} \mathrm{O}, \mathrm{R}_{f} 0.38\right)$ to give $46\left(1.24 \mathrm{~g}, 70 \%\right.$ ) as a yellow solid. $\mathrm{mp} 57-59^{\circ} \mathrm{C} ; v_{\max }\left(\right.$ film, $\left.\mathrm{cm}^{-1}\right) 1717$, 
1667, 1624, 1464, 1364, 1304, 1246, 1175, $1123 ;{ }^{1} \mathrm{H}$ NMR $\left(300 \mathrm{MHz}, \mathrm{CDCl}_{3}\right) \delta_{\mathrm{H}}: 1.38(3 \mathrm{H}, \mathrm{t}$, $\left.J 7.2 \mathrm{CH}_{3}\right), 4.33$ (2H. q. J 7.2, $\left.\mathrm{OCH}_{2}\right), 6.96\left(1 \mathrm{H}, \mathrm{d}, J 15.5, \mathrm{EtO}_{2} \mathrm{CCH}\right), 7.52-7.68(2 \mathrm{H}, \mathrm{m}, \mathrm{Ar} H)$, 7.86-8.10 (4H, m, ArH), 8.09 (1H, d, J 15.5, CHCOAr), 8.46-8.55 (1H, m, ArH); ${ }^{13} \mathrm{C}\left\{{ }^{1} \mathrm{H}\right\}$ NMR $\left(75 \mathrm{MHz}, \mathrm{CDCl}_{3}\right) \delta_{\mathrm{C}}: 14.4\left(\mathrm{CH}_{3}\right), 61.6\left(\mathrm{OCH}_{2}\right), 124.2(\mathrm{ArC}), 127.2(\mathrm{ArC}), 128.0(\mathrm{ArC})$, $129.1(\mathrm{ArC}), 129.2(\mathrm{ArC}), 129.9(\mathrm{ArC}), 131.3(\mathrm{ArC}), 132.6\left(\mathrm{C}=\mathrm{CCO}_{2}\right), 132.7(\mathrm{ArC}), 134.2$ $(\mathrm{ArC}), 136.0(\mathrm{ArC}), 136.6(\mathrm{C}=\mathrm{CCO}), 165.9\left(\mathrm{CO}_{2}\right), 189.4(\mathrm{CO})$; HRMS $\left(\mathrm{NSI}^{+}\right) \mathrm{C}_{16} \mathrm{H}_{15} \mathrm{O}_{3}$ $[\mathrm{M}+\mathrm{H}]^{+}$found 255.1017, requires $255.1016(+0.5 \mathrm{ppm})$.

\subsection{General procedure for the NHC-catalysed redox hetero-Diels-Alder reaction with $\alpha, \beta$-unsaturated $\gamma$-ketoesters}

The appropriate $\alpha$-aroyloxyaldehyde (1.5 eq), $\alpha, \beta$-unsaturated $\gamma$-ketoester (1.0 eq) and NHC precatalyst 2 ( $5 \mathrm{~mol} \%)$ were dissolved in anhydrous THF $(0.075 \mathrm{M})$ in a sealed vial containing $3 \AA$ molecular sieves. $\mathrm{Et}_{3} \mathrm{~N}$ (1.5 eq.) was added and the reaction stirred until complete by TLC analysis. The mixture was diluted with EtOAc and washed successively with $1 \mathrm{M} \mathrm{HCl}$, saturated $\mathrm{NaHCO}_{3}$ and brine. The organic layer was dried with $\mathrm{MgSO}_{4}$, filtered and concentrated under reduced pressure to give the crude product, which was purified by column chromatography.

\subsection{1 (3S,4S)-Ethyl 3-butyl-2-oxo-6-phenyl-3,4-dihydro-2H-pyran-4-carboxylate 20}

Following General Procedure 4.5, 1-oxohexan-2-yl 4-nitrobenzoate (200 mg, 0.75 mmol), (E)-ethyl 4-oxo-4-phenylbut-2-enoate $(102 \mathrm{mg}, 0.500 \mathrm{mmol})$, NHC precatalyst 2 (18.4 $\mathrm{mg}$, $50.0 \mu \mathrm{mol}), \mathrm{Et}_{3} \mathrm{~N}(105 \mu \mathrm{L}, 0.75 \mathrm{mmol})$ and THF $(10 \mathrm{~mL})$ were reacted for $5 \mathrm{~h}$. The crude $(>95: 5$ dr) was purified by column chromatography (95:5 petrol : EtOAc) to give $\mathbf{2 0}$ (129 mg, 85\%) as colourless solid. mp $61-63{ }^{\circ} \mathrm{C} ;[\alpha]_{\mathrm{D}}^{20}+185.5\left(c\right.$ 0.5, $\left.\mathrm{CHCl}_{3}\right)$; Chiral HPLC analysis, Chiralcel OJ-H (95:5 hexane : IPA, flow rate $\left.1 \mathrm{mLmin}^{-1}, 254 \mathrm{~nm}, 30^{\circ} \mathrm{C}\right) \mathrm{t}_{\mathrm{R}}(3 S, 4 S): 17.2 \mathrm{~min}, \mathrm{t}_{\mathrm{R}}(3 R, 4 R)$ : $22.3 \mathrm{~min},>99: 1 \mathrm{er} ; v_{\max }$ (solid) $2953(\mathrm{C}-\mathrm{H}), 1761(\mathrm{C}=\mathrm{O}), 1719(\mathrm{C}=\mathrm{O})$; ${ }^{1} \mathrm{H}$ NMR $(400 \mathrm{MHz}$, $\left.\mathrm{CDCl}_{3}\right) \delta_{\mathrm{H}}: 0.91\left(3 \mathrm{H}, \mathrm{t}, J 7.1, \mathrm{CH}_{2} \mathrm{CH}_{3}\right), 1.25\left(3 \mathrm{H}, \mathrm{t}, J 7.1, \mathrm{OCH}_{2} \mathrm{CH}_{3}\right), 1.31-1.54(5 \mathrm{H}, \mathrm{m}$, $\left(\mathrm{CH}_{2}\right)_{2}$ and $\left.\mathrm{C}(3) \mathrm{CH}^{\mathrm{A}} \mathrm{H}^{\mathrm{B}}\right), 1.99-2.13\left(1 \mathrm{H}, \mathrm{m}, \mathrm{C}(3) \mathrm{CH}^{\mathrm{A}} H^{\mathrm{B}}\right), 2.68-2.79(1 \mathrm{H}, \mathrm{m}, \mathrm{C}(3) H), 3.53$ $(1 \mathrm{H}, \mathrm{t}, J 6.3, \mathrm{C}(4) H), 4.17\left(2 \mathrm{H}, \mathrm{qd}, J 7.1,2.5, \mathrm{OCH}_{2} \mathrm{CH}_{3}\right), 5.88(1 \mathrm{H}, \mathrm{d}, J 6.5, \mathrm{C}(5) H), 7.32-$ $7.41(3 \mathrm{H}, \mathrm{m}, \operatorname{ArC}(2) H$ and $\operatorname{ArC}(4) H), 7.61-7.66(2 \mathrm{H}, \mathrm{m}, \operatorname{ArC}(3) H) ;{ }^{13} \mathrm{C}\left\{{ }^{1} \mathrm{H}\right\} \mathrm{NMR}(100 \mathrm{MHz}$, $\left.\mathrm{CDCl}_{3}\right) \delta_{\mathrm{C}}: 12.8\left(\mathrm{CH}_{2} \mathrm{CH}_{3}\right), 13.0\left(\mathrm{OCH}_{2} \mathrm{CH}_{3}\right), 21.5\left(\mathrm{CH}_{2}\right), 25.8\left(\mathrm{CH}_{2}\right), 28.3\left(\mathrm{CH}_{2}\right), 39.5(\mathrm{C}(3))$, $40.8(C(4)), 60.5\left(\mathrm{OCH}_{2} \mathrm{CH}_{3}\right), 96.9(C(5)), 123.8(\mathrm{ArC}(3)), 127.5(\mathrm{ArC}(2)), 128.4(\mathrm{ArC}(4))$, 130.9 (ArC(1)), $151.1(C(6)), 168.2(C(2)), 169.2\left(C_{2} \mathrm{Et}\right)$; HRMS (NSI $\left.{ }^{+}\right) \mathrm{C}_{18} \mathrm{H}_{26} \mathrm{O}_{4} \mathrm{~N}$ $\left[\mathrm{M}+\mathrm{NH}_{4}\right]^{+}$found 320.1864 , requires $320.1856(+2.4 \mathrm{ppm})$. 


\subsection{2 (3S,4S)-Ethyl 3-benzyl-2-oxo-6-phenyl-3,4-dihydro-2H-pyran-4-carboxylate 21}

Following General Procedure 4.5, 1-oxo-3-phenylpropan-2-yl 4-nitrobenzoate 7 (238 mg, $0.80 \mathrm{mmol}),(E)$-ethyl 4-oxo-4-phenylbut-2-enoate (108 $\mathrm{mg}, 0.53 \mathrm{mmol})$, NHC precatalyst 2 $(19.5 \mathrm{mg}, 53 \mu \mathrm{mol}), \mathrm{Et}_{3} \mathrm{~N}(0.11 \mathrm{~mL}, 0.80 \mathrm{mmol})$ and THF $(5 \mathrm{~mL})$ were reacted for $2 \mathrm{~h}$. The crude ( $>95: 5 \mathrm{dr}$ ) was purified by column chromatography (95:5 petrol : EtOAc) to give 21 (140 $\mathrm{mg}, 79 \%$ ) as a colourless oil, with spectroscopic data in accordance with the literature. ${ }^{6 \mathrm{c}}[\alpha]_{\mathrm{D}}^{20}$ $+173.0\left(c\right.$ 1.05, $\left.\mathrm{CHCl}_{3}\right)\left\{\right.$ Lit. $^{6 \mathrm{c}}+107.2\left(c\right.$ 0.82, $\left.\left.\mathrm{CHCl}_{3}\right)\right\}$; Chiral HPLC analysis Chiralpak AD$\mathrm{H}\left(90: 10\right.$ hexane : IPA, flow rate $\left.1.0 \mathrm{mLmin}^{-1}, 254 \mathrm{~nm}, 30{ }^{\circ} \mathrm{C}\right)>99: 1 \mathrm{er} ;{ }^{1} \mathrm{H}$ NMR $(300 \mathrm{MHz}$, $\left.\mathrm{CDCl}_{3}\right) \delta_{\mathrm{H}}: 1.29\left(3 \mathrm{H}, \mathrm{t}, J 7.1, \mathrm{OCH}_{2} \mathrm{CH}_{3}\right) 2.77\left(1 \mathrm{H}, \mathrm{dd}, J 14.2,10.2, \mathrm{PhCH} H^{\mathrm{A}} \mathrm{H}^{\mathrm{B}}\right), 2.97-3.03$ $(1 \mathrm{H}, \mathrm{m}, \mathrm{C}(3) H), 3.25(1 \mathrm{H}, \mathrm{dd}, J 6.9,5.9, \mathrm{C}(4) H) 3.55\left(1 \mathrm{H}, \mathrm{dd}, J 14.2,4.6, \mathrm{PhCH}^{\mathrm{A}} H^{\mathrm{B}}\right), 4.22$ $\left(2 \mathrm{H}, \mathrm{q}, J 7.1, \mathrm{OCH}_{2} \mathrm{CH}_{3}\right), 5.80(1 \mathrm{H}, \mathrm{d}, J 7.0, \mathrm{C}(5) H), 7.16-7.21(2 \mathrm{H}, \mathrm{m}, \mathrm{ArH}), 7.26-7.40(6 \mathrm{H}$, m, ArH), 7.60-7.63 (2H, m, ArH).

\subsection{3 (3S,4S)-Ethyl 3-(2-(benzyloxy)ethyl)-2-oxo-6-phenyl-3,4-dihydro-2H-pyran-4-}

\section{carboxylate 22}

Following General Procedure 4.5, 4-(benzyloxy)-1-oxobutan-2-yl 4-nitrobenzoate (257 mg, $0.75 \mathrm{mmol}),(E)$-ethyl 4-oxo-4-phenylbut-2-enoate (102 $\mathrm{mg}, 0.50 \mathrm{mmol})$, NHC precatalyst 2 (9.2 mg, $25.0 \mu \mathrm{mol}), \mathrm{Et}_{3} \mathrm{~N}(105 \mu \mathrm{L}, 0.75 \mathrm{mmol})$ and THF $(5 \mathrm{~mL})$ were reacted for $9 \mathrm{~h}$. The crude $(90: 10 \mathrm{dr})$ was purified by column chromatography $\left(85: 15\right.$ petrol : EtOAc, $\left.\mathrm{R}_{f} 0.25\right)$ to give $22(161 \mathrm{mg}, 85 \%)$ as white solid. $\mathrm{mp} 77-78^{\circ} \mathrm{C}$ (hexane); $[\alpha]_{\mathrm{D}}^{20}+169.1\left(c 0.55\right.$ in $\left.\mathrm{CHCl}_{3}\right)$; Chiral HPLC analysis, Chiralcel OD-H (95:5 hexane : IPA, flow rate $1 \mathrm{mLmin}^{-1}, 254 \mathrm{~nm}, 30$ $\left.{ }^{\circ} \mathrm{C}\right) \mathrm{t}_{\mathrm{R}}(3 R, 4 R): 23.2 \mathrm{~min}, \mathrm{t}_{\mathrm{R}}(3 S, 4 S): 25.1 \mathrm{~min},>99: 1 \mathrm{er} ; v_{\max }\left(\right.$ film, $\left.\mathrm{cm}^{-1}\right) 1767(\mathrm{C}=\mathrm{O}), 1715$ $(\mathrm{C}=\mathrm{O}), 1657(\mathrm{C}=\mathrm{C}) ;{ }^{1} \mathrm{H}$ NMR $\left(300 \mathrm{MHz}, \mathrm{CDCl}_{3}\right) \delta_{\mathrm{H}}: 1.23\left(3 \mathrm{H}, \mathrm{t}, J 7.1, \mathrm{OCH}_{2} \mathrm{CH}_{3}\right), 1.72-1.83$ $\left(1 \mathrm{H}, \mathrm{m}, \mathrm{C}(3) \mathrm{C}^{\mathrm{A}} \mathrm{H}^{\mathrm{B}}\right), 2.37-2.48\left(1 \mathrm{H}, \mathrm{m}, \mathrm{C}(3) \mathrm{CH}^{\mathrm{A}} H^{\mathrm{B}}\right), 3.04(1 \mathrm{H}, \mathrm{q}, J 6.7, \mathrm{C}(3) H), 3.50(1 \mathrm{H}, \mathrm{t}$, $J$ 6.5, C(4)H), 3.60-3.72 (2H, m, C(3) $\left.\mathrm{CH}_{2} \mathrm{CH}_{2}\right), 4.09-4.20\left(2 \mathrm{H}, \mathrm{m}, \mathrm{OCH}_{2} \mathrm{CH}_{3}\right), 4.50(2 \mathrm{H}, \mathrm{d}, J$ 2.1, $\left.\mathrm{OCH}_{2} \mathrm{Ar}\right), 5.86(1 \mathrm{H}, \mathrm{d}, J 6.9, \mathrm{C}(5) H), 7.27-7.40(8 \mathrm{H}, \mathrm{m}, \mathrm{ArH}), 7.61-7.66(2 \mathrm{H}, \mathrm{m}, \mathrm{Ar} H)$; ${ }^{13} \mathrm{C}\left\{{ }^{1} \mathrm{H}\right\}$ NMR $\left(75 \mathrm{MHz}, \mathrm{CDCl}_{3}\right) \delta_{\mathrm{C}}: 14.2\left(\mathrm{CH}_{3}\right), 27.8\left(\mathrm{CH}_{2}\right), 37.6(C(3) \mathrm{H}), 42.1(C(4) \mathrm{H}), 61.7$ $\left(\mathrm{OCH}_{2} \mathrm{CH}_{3}\right), 67.4\left(\mathrm{OCH}_{2} \mathrm{CH}_{2}\right), 73.2\left(\mathrm{OCH}_{2} \mathrm{Ph}\right), 98.2(\mathrm{C}(5) \mathrm{H}), 125.0(\mathrm{ArCH}), 127.8(\mathrm{ArCH})$, $128.5(\mathrm{ArCH}), 128.6(\mathrm{ArCH}), 129.6(\mathrm{ArCH}), 132.0(\mathrm{ArC}), 138.2(\mathrm{ArC}), 152.4(C(6)), 169.4$ $(C(2)), 170.4\left(\mathrm{CO}_{2} \mathrm{Et}\right)$; HRMS $\left(\mathrm{NSI}^{+}\right) \mathrm{C}_{23} \mathrm{H}_{25} \mathrm{O}_{5}[\mathrm{M}+\mathrm{H}]^{+}$found 381.1697, requires 381.1697 $(+0.1 \mathrm{ppm})$. 


\subsection{4 (3S,4S)-Ethyl 3-benzyl-6-methyl-2-oxo-3,4-dihydro-2H-pyran-4-carboxylate 23}

In a modified procedure, 1-oxo-3-phenylpropan-2-yl 4-nitrobenzoate 7 (196 mg, $0.65 \mathrm{mmol}$ ), (E)-ethyl 4-oxopent-2-enoate 41 (62 mg, $0.44 \mathrm{mmol}$ ), NHC precatalyst 2 ( $8.0 \mathrm{mg}, 21.8 \mu \mathrm{mol})$, and $\mathrm{Cs}_{2} \mathrm{CO}_{3}(142 \mathrm{mg}, 0.44 \mathrm{mmol})$ were dissolved in THF $(4.4 \mathrm{~mL})$ in a sealed vial and heated at $40{ }^{\circ} \mathrm{C}$ for $7 \mathrm{~h}$. The mixture was then diluted with EtOAc and washed successively with $1 \mathrm{M}$ $\mathrm{HCl}(\times 2), \mathrm{NaHCO}_{3}(\times 2)$, and brine $(\times 2)$ before being dried over $\mathrm{MgSO}_{4}$ and concentrated under reduced pressure. The crude $(>95: 5 \mathrm{dr})$ was purified by column chromatography $(95: 5$ petrol : EtOAc, $\mathrm{R}_{f} 0.20$ ) to give 23 ( $79 \mathrm{mg}, 65 \%$ ) as white solid, with spectroscopic data in accordance to the literature. ${ }^{6 \mathrm{c}} \mathrm{mp} 88-89{ }^{\circ} \mathrm{C}$ (hexane); $[\alpha]_{\mathrm{D}}^{20}+270.9\left(c \quad 0.32\right.$ in $\left.\mathrm{CHCl}_{3}\right)\left\{\right.$ Lit. $^{6 \mathrm{c}}+250.6(c$ 0.93, $\left.\left.\mathrm{CHCl}_{3}\right)\right\}$; Chiral HPLC analysis, Chiralcel AD-H (95:5 hexane : IPA, flow rate $1 \mathrm{mLmin}^{-}$ $\left.{ }^{1}, 254 \mathrm{~nm}, 30^{\circ} \mathrm{C}\right) \mathrm{t}_{\mathrm{R}}(3 R, 4 R): 11.0 \mathrm{~min}, \mathrm{t}_{\mathrm{R}}(3 S, 4 S): 13.2 \mathrm{~min}, 98.5: 1.5 \mathrm{er} ; v_{\max }\left(\right.$ film, $\left.\mathrm{cm}^{-1}\right) 1771$ $(\mathrm{C}=\mathrm{O}), 1726(\mathrm{C}=\mathrm{O}), 1688(\mathrm{C}=\mathrm{C}) ;{ }^{1} \mathrm{H} \mathrm{NMR}\left(300 \mathrm{MHz}, \mathrm{CDCl}_{3}\right) \delta_{\mathrm{H}}: 1.21(3 \mathrm{H}, \mathrm{t}, J$ 7.1, $\left.\mathrm{OCH}_{2} \mathrm{CH}_{3}\right), 1.83\left(3 \mathrm{H}, \mathrm{s}, \mathrm{C}(6) \mathrm{CH}_{3}\right), 2.61\left(1 \mathrm{H}, \mathrm{dd}, J 14.3,10.1, \mathrm{C}(3) \mathrm{CH}^{\mathrm{A}} \mathrm{H}^{\mathrm{B}}\right), 2.75-2.82(1 \mathrm{H}$, m, C(3)H), $2.92(1 \mathrm{H}, \mathrm{t}, J 6.4, \mathrm{C}(4) H), 3.42\left(1 \mathrm{H}, \mathrm{dd}, J 14.2,4.6, \mathrm{C}(3) \mathrm{CH}^{\mathrm{A}} H^{\mathrm{B}}\right), 4.13(2 \mathrm{H}, \mathrm{q}, J$ 7.2, $\left.\mathrm{OCH}_{2}\right), 4.95(1 \mathrm{H}, \mathrm{dd}, J 6.8,1.1, \mathrm{C}(5) H), 7.04-7.10(2 \mathrm{H}, \mathrm{m}, \operatorname{ArC}(2) H), 7.12-7.27(3 \mathrm{H}, \mathrm{m}$, $\operatorname{ArC}(3) H$ and $\operatorname{ArC}(4) H) ;{ }^{13} \mathrm{C}\left\{{ }^{1} \mathrm{H}\right\} \operatorname{NMR}\left(75 \mathrm{MHz}, \mathrm{CDCl}_{3}\right) \delta_{\mathrm{C}}: 14.2\left(\mathrm{CH}_{2} \mathrm{CH}_{3}\right), 18.9\left(\mathrm{CCH}_{3}\right)$, $33.3\left(\mathrm{CH}_{2}\right), 40.4(C(3) \mathrm{H}), 42.8(C(4) \mathrm{H}), 61.5\left(\mathrm{OCH}_{2}\right), 98.7(C(5) \mathrm{H}), 126.9(\mathrm{ArCH}), 128.8$ $(\mathrm{ArCH}), 129.1(\mathrm{ArCH}), 138.3(\mathrm{ArC}), 152.4(C(6)), 169.3(C(2)), 170.7\left(\mathrm{CO}_{2} \mathrm{Et}\right) ; \mathrm{HRMS}\left(\mathrm{NSI}^{+}\right)$ $\mathrm{C}_{16} \mathrm{H}_{19} \mathrm{O}_{4}[\mathrm{M}+\mathrm{H}]^{+}$found 275.1280, requires 275.1278 (+0.8 ppm).

\subsection{5 (3S,4S)-Ethyl 3-butyl-2-oxo-6-(p-tolyl)-3,4-dihydro-2H-pyran-4-carboxylate 24}

Following General Procedure 4.5, 1-oxohexan-2-yl 4-nitrobenzoate (200 mg, $0.75 \mathrm{mmol}$ ), (E)-ethyl 4-oxo-4-( $p$-tolyl)but-2-enoate $42(109 \mathrm{mg}, 0.50 \mathrm{mmol})$, NHC precatalyst $2(9.2 \mathrm{mg}$, $25.0 \mu \mathrm{mol}), \mathrm{Et}_{3} \mathrm{~N}(105 \mu \mathrm{L}, 0.75 \mathrm{mmol})$ and THF $(5 \mathrm{~mL})$ were reacted for $6 \mathrm{~h}$. The crude $(>95: 5$ dr) was purified by column chromatography (90:10 hexane : $\left.\mathrm{Et}_{2} \mathrm{O}, \mathrm{R}_{f} 0.19\right)$ to give $24(127 \mathrm{mg}$, $80 \%$ ) as colourless needles. mp $64-65^{\circ} \mathrm{C}$ (hexane); $[\alpha]_{\mathrm{D}}^{20}+158.4\left(c 0.38\right.$ in $\left.\mathrm{CHCl}_{3}\right)$; Chiral HPLC analysis, Chiralcel OD-H (95:5 hexane : IPA, flow rate $\left.1 \mathrm{mLmin}^{-1}, 254 \mathrm{~nm}, 30{ }^{\circ} \mathrm{C}\right) \mathrm{t}_{\mathrm{R}}$ $(3 S, 4 S): 8.3 \mathrm{~min}, \mathrm{t}_{\mathrm{R}}(3 R, 4 R): 9.0 \mathrm{~min},>99: 1 \mathrm{er} ; v_{\max }\left(\right.$ film, $\left.\mathrm{cm}^{-1}\right) 1763(\mathrm{C}=\mathrm{O}), 1719(\mathrm{C}=\mathrm{O})$, $1684(\mathrm{C}=\mathrm{C}) ;{ }^{1} \mathrm{H}$ NMR $\left(300 \mathrm{MHz}, \mathrm{CDCl}_{3}\right) \delta_{\mathrm{H}}: 0.91\left(3 \mathrm{H}, \mathrm{t}, J 7.1,\left(\mathrm{CH}_{2}\right)_{3} \mathrm{CH}_{3}\right), 1.25(3 \mathrm{H}, \mathrm{t}, J 7.2$, $\left.\mathrm{OCH}_{2} \mathrm{CH}_{3}\right), 1.31-1.52\left(5 \mathrm{H}, \mathrm{m}, \mathrm{C}(3) H_{\mathrm{a}} \mathrm{H}_{\mathrm{b}}\right.$ and $\left.\mathrm{CH}_{2} \times 2\right), 1.98-2.15\left(1 \mathrm{H}, \mathrm{m}, \mathrm{C}(3) \mathrm{H}_{\mathrm{a}} H_{\mathrm{b}}\right), 2.36$ $\left(3 \mathrm{H}, \mathrm{s}, \mathrm{ArC}(4) \mathrm{CH}_{3}\right), 2.73(1 \mathrm{H}, \mathrm{q}, J 6.5, \mathrm{C}(3) H), 3.51(1 \mathrm{H}, \mathrm{t}, J 6.3, \mathrm{C}(4) H), 4.17(2 \mathrm{H}, \mathrm{qd}, J 7.2$, 1.9, $\left.\mathrm{OCH}_{2}\right), 5.82(1 \mathrm{H}, \mathrm{d}, J 6.5, \mathrm{C}(5) H), 7.18(2 \mathrm{H}, \mathrm{d}, J 8.1, \operatorname{ArC}(3) H), 7.53(2 \mathrm{H}, \mathrm{d}, J 8.3$, $\operatorname{ArC}(2) H) ;{ }^{13} \mathrm{C}\left\{{ }^{1} \mathrm{H}\right\}$ NMR $\left(75 \mathrm{MHz}, \mathrm{CDCl}_{3}\right) \delta_{\mathrm{C}}: 14.0\left(\mathrm{CH}_{3}\right), 14.2\left(\mathrm{CH}_{3}\right), 21.4\left(\mathrm{CH}_{3}\right), 22.6$ 
$\left(\mathrm{CH}_{2}\right), 26.9\left(\mathrm{CH}_{2}\right), 29.5\left(\mathrm{CH}_{2}\right), 40.7(C(3) \mathrm{H}), 42.0(C(4) \mathrm{H}), 61.6\left(\mathrm{OCH}_{2}\right), 97.1(C(5) \mathrm{H}), 124.9$ $(\mathrm{ArC}), 129.2(\mathrm{ArCH}), 129.3(\mathrm{ArCH}), 139.7(\mathrm{ArC}), 152.4(C(6)), 169.6(C(2)), 170.5\left(\mathrm{CO}_{2} \mathrm{Et}\right)$; HRMS $\left(\mathrm{NSI}^{+}\right) \mathrm{C}_{19} \mathrm{H}_{25} \mathrm{O}_{4}[\mathrm{M}+\mathrm{H}]^{+}$found 317.1751, requires 317.1747 (+1.1 ppm).

\subsection{6 (3S,4S)-Ethyl 3-butyl-6-(4-methoxyphenyl)-2-oxo-3,4-dihydro-2H-pyran-4- carboxylate 25}

Following General Procedure 4.5, 1-oxohexan-2-yl 4-nitrobenzoate (200 mg, 0.75 mmol), (E)-ethyl 4-(4-methoxyphenyl)-4-oxobut-2-enoate 43 (117 mg, $0.50 \mathrm{mmol})$, NHC precatalyst $2(9.2 \mathrm{mg}, 25.0 \mu \mathrm{mol}), \mathrm{Et}_{3} \mathrm{~N}(105 \mu \mathrm{L}, 0.75 \mathrm{mmol})$ and THF $(5 \mathrm{~mL})$ were reacted for $6 \mathrm{~h}$. The crude ( $>95: 5 \mathrm{dr}$ ) was purified by column chromatography $\left(85: 15\right.$ hexane : $\left.\mathrm{Et}_{2} \mathrm{O}, \mathrm{R}_{f} 0.17\right)$ to give 25 (149 mg, 90\%) as white solid. $\mathrm{mp} 61-62{ }^{\circ} \mathrm{C}$ (hexane); $[\alpha]_{\mathrm{D}}^{20}+123.6\left(c 0.39\right.$ in $\left.\mathrm{CHCl}_{3}\right)$; Chiral HPLC analysis, Chiralcel OD-H (95:5 hexane : IPA, flow rate $1 \mathrm{mLmin}^{-1}, 254 \mathrm{~nm}, 30$ $\left.{ }^{\circ} \mathrm{C}\right) \mathrm{t}_{\mathrm{R}}(3 R, 4 R): 13.9 \mathrm{~min}, \mathrm{t}_{\mathrm{R}}(3 S, 4 S): 14.5 \mathrm{~min},>99: 1 \mathrm{er} ; v_{\max }\left(\right.$ film, $\left.\mathrm{cm}^{-1}\right) 1751(\mathrm{C}=\mathrm{O}), 1726$ $(\mathrm{C}=\mathrm{O}), 1661(\mathrm{C}=\mathrm{C}) ;{ }^{1} \mathrm{H}$ NMR $\left(300 \mathrm{MHz}, \mathrm{CDCl}_{3}\right) \delta_{\mathrm{H}}: 0.91\left(3 \mathrm{H}, \mathrm{t}, J 7.1, \mathrm{CH}_{2} \mathrm{CH}_{3}\right), 1.25(3 \mathrm{H}, \mathrm{t}$, $\left.J 7.2, \mathrm{OCH}_{2} \mathrm{CH}_{3}\right), 1.31-1.49\left(5 \mathrm{H}, \mathrm{m}, \mathrm{C}(3) H_{\mathrm{a}} \mathrm{H}_{\mathrm{b}}\right), 1.99-2.11\left(1 \mathrm{H}, \mathrm{m}, \mathrm{C}(3) \mathrm{H}_{\mathrm{a}} H_{\mathrm{b}}\right), 2.72(1 \mathrm{H}, \mathrm{q}, J$ 6.5, C(3)H), $3.50\left(1 \mathrm{H}, \mathrm{t}, J\right.$ 6.3, C(4)H), $3.82\left(3 \mathrm{H}, \mathrm{s}, \operatorname{ArC}(4) \mathrm{OCH}_{3}\right), 4.17(2 \mathrm{H}, \mathrm{qd}, J$ 7.1, 1.9, $\left.\mathrm{OCH}_{2}\right), 5.74(1 \mathrm{H}, \mathrm{d}, J 6.5, \mathrm{C}(5) H), 6.89(2 \mathrm{H}, \mathrm{d}, J 8.9, \operatorname{ArC}(3) H), 7.57(2 \mathrm{H}, \mathrm{d}, J 9.0, \operatorname{ArC}(2) H)$; ${ }^{13} \mathrm{C}\left\{{ }^{1} \mathrm{H}\right\}$ NMR $\left(75 \mathrm{MHz}, \mathrm{CDCl}_{3}\right) \delta_{\mathrm{C}}: 14.0\left(\mathrm{CH}_{3}\right), 14.2\left(\mathrm{CH}_{3}\right), 22.6\left(\mathrm{CH}_{2}\right), 26.9\left(\mathrm{CH}_{2}\right), 29.5$ $\left(\mathrm{CH}_{2}\right), 40.8(C(3) \mathrm{H}), 41.9(C(4) \mathrm{H}), 55.5\left(\mathrm{OCH}_{3}\right), 61.6\left(\mathrm{OCH}_{2}\right), 96.0(C(5) \mathrm{H}), 114.0(\mathrm{ArC})$, 124.6 (ArCH), $126.4(\mathrm{ArCH}), 152.1(C(6)), 160.7$ (ArC), $169.6(C(2)), 170.6\left(C_{2} \mathrm{Et}\right)$; HRMS $\left(\mathrm{NSI}^{+}\right) \mathrm{C}_{19} \mathrm{H}_{25} \mathrm{O}_{5}[\mathrm{M}+\mathrm{H}]^{+}$found 333.1699, requires 333.1697 (+0.7 ppm).

\subsection{7 (3S,4S)-Ethyl 6-(4-chlorophenyl)-3-methyl-2-oxo-3,4-dihydro-2H-pyran-4- carboxylate 26}

Following General Procedure 4.5, 1-oxopropan-2-yl 4-nitrobenzoate (167 mg, $0.75 \mathrm{mmol}$ ), (E)-ethyl 4-(4-chlorophenyl)-4-oxobut-2-enoate 44 (119 $\mathrm{mg}, 0.50 \mathrm{mmol})$, NHC precatalyst 2 (9.2 mg, $25.0 \mu \mathrm{mol}), \mathrm{Et}_{3} \mathrm{~N}(105 \mu \mathrm{L}, 0.75 \mathrm{mmol})$ and THF $(5 \mathrm{~mL})$ were reacted for $4 \mathrm{~h}$. The crude (80:20 dr) was purified by column chromatography (70:30 hexane : $\mathrm{Et}_{2} \mathrm{O}$, minor-anti $\mathrm{R}_{f}$ 0.29 , major-syn $\left.\mathrm{R}_{f} 0.23\right)$ to give $\mathbf{2 6}\left(81 \mathrm{mg}, 55 \%\right.$ ) as white solid. mp $93-95{ }^{\circ} \mathrm{C}$ (hexane); $[\alpha]_{\mathrm{D}}^{20}$ +248.5 (c 0.27 in $\mathrm{CHCl}_{3}$ ); Chiral HPLC analysis, Chiralcel OD-H (95:5 hexane : IPA, flow rate $\left.1 \mathrm{mLmin}^{-1}, 254 \mathrm{~nm}, 30^{\circ} \mathrm{C}\right) \mathrm{t}_{\mathrm{R}}(3 R, 4 R): 14.3 \mathrm{~min}, \mathrm{t}_{\mathrm{R}}(3 S, 4 S): 15.7 \mathrm{~min},>99: 1 \mathrm{er} ; v_{\max }(\mathrm{film}$, $\left.\mathrm{cm}^{-1}\right) 1765(\mathrm{C}=\mathrm{O}), 1721(\mathrm{C}=\mathrm{O}), 1657(\mathrm{C}=\mathrm{C}) ;{ }^{1} \mathrm{H}$ NMR $\left(300 \mathrm{MHz}, \mathrm{CDCl}_{3}\right) \delta_{\mathrm{H}}: 1.26(3 \mathrm{H}, \mathrm{t}, J$ 7.2, $\left.\mathrm{OCH}_{2} \mathrm{CH}_{3}\right), 1.34\left(3 \mathrm{H}, \mathrm{d}, J 7.0, \mathrm{C}(3) \mathrm{CH}_{3}\right), 2.92(1 \mathrm{H}$, pent, $J 6.7, \mathrm{C}(3) H), 3.44(1 \mathrm{H}, \mathrm{t}, J 6.3$, $\mathrm{C}(4) H), 4.19\left(2 \mathrm{H}, \mathrm{qd}, J 7.1,3.2, \mathrm{OCH}_{2}\right), 5.86(1 \mathrm{H}, \mathrm{d}, J 6.6, \mathrm{C}(5) H), 7.35(2 \mathrm{H}, \mathrm{d}, J 8.7$, 
$\operatorname{ArC}(2) H), 7.57(2 \mathrm{H}, \mathrm{d}, J$ 8.8, $\mathrm{ArC}(3) H) ;{ }^{13} \mathrm{C}\left\{{ }^{1} \mathrm{H}\right\} \mathrm{NMR}\left(75 \mathrm{MHz}, \mathrm{CDCl}_{3}\right) \delta_{\mathrm{C}}: 12.6\left(\mathrm{C}(3) \mathrm{CH}_{3}\right)$, $14.2\left(\mathrm{CH}_{2} \mathrm{CH}_{3}\right), 35.6(C(3) \mathrm{H}), 43.8(C(4) \mathrm{H}), 61.8\left(\mathrm{OCH}_{2}\right), 98.4(C(5) \mathrm{H}), 126.3(\mathrm{ArC}), 128.9$ $(\mathrm{ArCH}), 130.5(\mathrm{ArCH}), 135.6(\mathrm{ArC}), 151.6(C(6)), 169.6(C(2)), 170.2\left(\mathrm{CO}_{2} \mathrm{Et}\right) ; \mathrm{HRMS}\left(\mathrm{NSI}^{+}\right)$ $\mathrm{C}_{15} \mathrm{H}_{16} \mathrm{O}_{4} \mathrm{Cl}[\mathrm{N}+\mathrm{H}]^{+}$found 295.0735, requires 295.0732 (+1.1 ppm).

\subsection{8 (3S,4S)-Ethyl 3-benzyl-2-oxo-6-(4-(trifluoromethyl)phenyl)-3,4-dihydro-2H- pyran-4-carboxylate 27}

Following General Procedure 4.5, 1-oxo-3-phenylpropan-2-yl 4-nitrobenzoate 7 (224 mg, $0.75 \mathrm{mmol}$ ), (E)-ethyl 4-oxo-4-(4-(trifluoromethyl)phenyl)but-2-enoate 45 (136 mg, 0.50 mmol), NHC precatalyst $2(18.3 \mathrm{mg}, 50.0 \mu \mathrm{mol}), \mathrm{Et}_{3} \mathrm{~N}(105 \mu \mathrm{L}, 0.75 \mathrm{mmol})$ and THF $(5 \mathrm{~mL})$ were reacted for $3 \mathrm{~h}$. The crude $(80: 20 \mathrm{dr})$ was purified by column chromatography $(90: 10$ petrol : EtOAc, major-syn $\mathrm{R}_{f} 0.16$, minor-anti $\left.\mathrm{R}_{f} 0.09\right)$ to give 27 (105 mg, 52\%) as white solid. mp $145-147^{\circ} \mathrm{C}$ (hexane); $[\alpha]_{\mathrm{D}}^{20}+172.9$ ( $c 0.31$ in $\mathrm{CHCl}_{3}$ ); Chiral HPLC analysis, Chiralcel OD-H (95:5 hexane: IPA, flow rate $\left.1 \mathrm{mLmin}^{-1}, 254 \mathrm{~nm}, 30^{\circ} \mathrm{C}\right) \mathrm{t}_{\mathrm{R}}(3 S, 4 S): 18.8 \mathrm{~min}, \mathrm{t}_{\mathrm{R}}(3 R, 4 R)$ : $15.4 \mathrm{~min},>99: 1$ er; $v_{\max }\left(\right.$ film, $\left.\mathrm{cm}^{-1}\right) 1775(\mathrm{C}=\mathrm{O}), 1719(\mathrm{C}=\mathrm{O}), 1659(\mathrm{C}=\mathrm{C}) ;{ }^{1} \mathrm{H}$ NMR (300 $\left.\mathrm{MHz}, \mathrm{CDCl}_{3}\right) \delta_{\mathrm{H}}: 1.30\left(3 \mathrm{H}, \mathrm{t}, J 7.2, \mathrm{OCH}_{2} \mathrm{CH}_{3}\right), 2.78\left(1 \mathrm{H}, \mathrm{dd}, J 14.4,9.9, \mathrm{C}(3) \mathrm{H}_{\mathrm{a}} \mathrm{H}_{\mathrm{b}}\right), 2.98-$ $3.06(1 \mathrm{H}, \mathrm{m}, \mathrm{C}(3) H), 3.30(1 \mathrm{H}, \mathrm{t}, J 6.4, \mathrm{C}(4) H), 3.55\left(1 \mathrm{H}, \mathrm{dd}, J 14.2,4.7, \mathrm{C}(3) \mathrm{H}_{\mathrm{a}} H_{\mathrm{b}}\right), 4.23(2 \mathrm{H}$, q, $\left.J 7.2, \mathrm{OCH}_{2}\right), 5.92(1 \mathrm{H}, \mathrm{d}, J 7.0, \mathrm{C}(5) H), 7.17-7.19\left(2 \mathrm{H}, \mathrm{m}, \mathrm{C}(3) \mathrm{CH}_{2} \mathrm{ArC}(2) H\right), 7.24-7.36$ $\left(3 \mathrm{H}, \mathrm{m}, \mathrm{C}(3) \mathrm{CH}_{2} \mathrm{ArC}(3) \mathrm{H}\right.$ and $\left.\mathrm{C}(3) \mathrm{CH}_{2} \mathrm{ArC}(4) H\right), 7.63(2 \mathrm{H}, \mathrm{d}, J 8.5, \mathrm{C}(6) \mathrm{ArC}(2) H), 7.73(2 \mathrm{H}$, d, $J$ 8.5, C(6)ArC(3)H); ${ }^{13} \mathrm{C}\left\{{ }^{1} \mathrm{H}\right\} \operatorname{NMR}\left(75 \mathrm{MHz}, \mathrm{CDCl}_{3}\right) \delta_{\mathrm{C}}: 14.3\left(\mathrm{CH}_{3}\right), 33.3\left(\mathrm{CH}_{2} \mathrm{Ph}\right), 40.8$ $(C(3) \mathrm{H}), 42.8(C(4) \mathrm{H}), 62.0\left(\mathrm{OCH}_{2}\right), 100.3(C(5) \mathrm{H}), 124.0\left(\mathrm{q},{ }^{1} J_{\mathrm{CF}} 272, C \mathrm{~F}_{3}\right), 125.2(\mathrm{ArCH})$, $125.7\left(\mathrm{q},{ }^{3} J_{\mathrm{CF}} 3.8, \mathrm{C}(6) \operatorname{ArC}(3,5) \mathrm{H}\right), 127.1(\mathrm{ArCH}), 129.0(\mathrm{ArCH}), 129.1(\mathrm{ArCH}), 131.4\left(\mathrm{q},{ }^{2} J_{\mathrm{CF}}\right.$ 32.5, C(6)ArC(4)), $135.2(\mathrm{ArC}), 137.9(\mathrm{ArC}), 151.4(C(6)), 168.4(C(2)), 170.0\left(\mathrm{CO}_{2} \mathrm{Et}\right)$; $\mathrm{HRMS}\left(\mathrm{NSI}^{+}\right) \mathrm{C}_{22} \mathrm{H}_{23} \mathrm{O}_{4} \mathrm{NF}_{3}\left[\mathrm{M}+\mathrm{NH}_{4}\right]^{+}$found 422.1574, requires 422.1574 (+0.1 ppm).

\subsection{9 (3S,4S)-Ethyl 3-methyl-6-(naphthalen-2-yl)-2-oxo-3,4-dihydro-2H-pyran-4- carboxylate 28 and $(3 S, 4 R)$-Ethyl 3-methyl-6-(naphthalen-2-yl)-2-oxo-3,4-dihydro-2H- pyran-4-carboxylate 29}

Following General Procedure 4.5, 1-oxopropan-2-yl 4-nitrobenzoate (167 mg, 0.75 mmol), (E)-ethyl 4-(naphthalen-2-yl)-4-oxobut-2-enoate 46 (127 mg, $0.50 \mathrm{mmol})$, NHC precatalyst 2 (9.2 mg, $25.0 \mu \mathrm{mol}), \mathrm{Et}_{3} \mathrm{~N}(105 \mu \mathrm{L}, 0.75 \mathrm{mmol})$ and THF $(5 \mathrm{~mL})$ were reacted for $24 \mathrm{~h}$. The crude (50:50 dr) was purified by column chromatography (90:10 petrol : EtOAc, anti $\mathrm{R}_{f}$ 0.29, syn $\mathrm{R}_{f} 0.23$ ) to give syn-28 (49 mg, 32\%) as white solid and anti-29 (55 mg, 35\%) as white solid. syn-28: $\mathrm{mp} 101-103{ }^{\circ} \mathrm{C}$ (hexane); $[\alpha]_{\mathrm{D}}^{20}+220.3$ (c 0.34 in $\left.\mathrm{CHCl}_{3}\right)$; Chiral HPLC analysis, 
Chiralcel OD-H (95:5 hexane : IPA, flow rate $\left.1 \mathrm{mLmin}^{-1}, 254 \mathrm{~nm}, 30^{\circ} \mathrm{C}\right) \mathrm{t}_{\mathrm{R}}(3 S, 4 S): 26.7 \mathrm{~min}$, $\mathrm{t}_{\mathrm{R}}(3 R, 4 R): 24.1 \mathrm{~min},>99: 1 \mathrm{er} ; v_{\max }\left(\right.$ film, $\left.\mathrm{cm}^{-1}\right) 1771(\mathrm{C}=\mathrm{O}), 1715(\mathrm{C}=\mathrm{O}), 1651(\mathrm{C}=\mathrm{C}) ;{ }^{1} \mathrm{H}$ $\mathrm{NMR}\left(300 \mathrm{MHz}, \mathrm{CDCl}_{3}\right) \delta_{\mathrm{H}}: 1.27\left(3 \mathrm{H}, \mathrm{t}, J 7.2, \mathrm{OCH}_{2} \mathrm{CH}_{3}\right), 1.37\left(3 \mathrm{H}, \mathrm{d}, J 6.9, \mathrm{C}(3) \mathrm{CH}_{3}\right), 2.97$ $(1 \mathrm{H}$, pent, $J 6.7, \mathrm{C}(3) H), 3.49(1 \mathrm{H}, \mathrm{t}, J 6.3, \mathrm{C}(4) H), 4.15-4.26\left(2 \mathrm{H}, \mathrm{m}, \mathrm{OCH}_{2}\right), 6.02(1 \mathrm{H}, \mathrm{d}, J$ 6.6, C(5)H), 7.48-7.53 (2H, m, $\operatorname{Ar} H), 7.65(1 \mathrm{H}, \mathrm{dd}, J 8.7,1.9, \operatorname{ArC}(2) H), 7.78-7.91(3 \mathrm{H}, \mathrm{m}$, $\mathrm{Ar} H), 8.16-8.22(1 \mathrm{H}, \mathrm{m}, \mathrm{Ar} H) ;{ }^{13} \mathrm{C}\left\{{ }^{1} \mathrm{H}\right\} \mathrm{NMR}\left(75 \mathrm{MHz}, \mathrm{CDCl}_{3}\right) \delta_{\mathrm{C}}: 12.7\left(\mathrm{C}(3) \mathrm{CH}_{3}\right), 14.2$ $\left(\mathrm{CH}_{2} \mathrm{CH}_{3}\right), 35.7(C(3) \mathrm{H}), 43.9(\mathrm{C}(4) \mathrm{H}), 61.8\left(\mathrm{OCH}_{2}\right), 98.5(\mathrm{C}(5) \mathrm{H}), 122.1(\mathrm{ArCH}), 124.6$ $(\mathrm{ArCH}), 126.8(\mathrm{ArCH}), 127.1(\mathrm{ArCH}), 127.7(\mathrm{ArCH}), 128.4(\mathrm{ArCH}), 128.8(\mathrm{ArCH}), 129.0$ $(\mathrm{ArC}), 133.1(\mathrm{ArC}), 133.7(\mathrm{ArC}), 152.4(C(6)), 170.0(C(2)), 170.3\left(C \mathrm{O}_{2} \mathrm{Et}\right) ; \mathrm{HRMS}\left(\mathrm{NSI}^{+}\right)$ $\mathrm{C}_{19} \mathrm{H}_{19} \mathrm{O}_{4}[\mathrm{M}+\mathrm{H}]^{+}$found 311.1282, requires 311.1278 (+1.3 ppm).

anti-29: mp 92-94 ${ }^{\circ} \mathrm{C}$ (hexane); $[\alpha]_{\mathrm{D}}^{20}-91.9$ ( $c 0.37$ in $\mathrm{CHCl}_{3}$ ); Chiral HPLC analysis, Chiralcel OD-H (95:5 hexane : IPA, flow rate $\left.1 \mathrm{mLmin}^{-1}, 254 \mathrm{~nm}, 30^{\circ} \mathrm{C}\right) \mathrm{t}_{\mathrm{R}}(3 R, 4 S): 15.2 \mathrm{~min}$, $\mathrm{t}_{\mathrm{R}}(3 S, 4 R): 15.9 \mathrm{~min},>99: 1 \mathrm{er} ; v_{\max }\left(\right.$ film, $\left.\mathrm{cm}^{-1}\right) 1769(\mathrm{C}=\mathrm{O}), 1728(\mathrm{C}=\mathrm{O}), 1661(\mathrm{C}=\mathrm{C}) ;{ }^{1} \mathrm{H}$ $\mathrm{NMR}\left(300 \mathrm{MHz}, \mathrm{CDCl}_{3}\right) \delta_{\mathrm{H}}: 1.33\left(3 \mathrm{H}, \mathrm{t}, J 7.2, \mathrm{OCH}_{2} \mathrm{CH}_{3}\right), 1.40\left(3 \mathrm{H}, \mathrm{d}, J 6.9, \mathrm{C}(3) \mathrm{CH}_{3}\right), 3.10$ $3.20(1 \mathrm{H}, \mathrm{m}, \mathrm{C}(3) H), 3.38(1 \mathrm{H}, \mathrm{dd}, J 9.1,4.0, \mathrm{C}(4) H), 4.27\left(2 \mathrm{H}, \mathrm{q}, J 7.2, \mathrm{OCH}_{2}\right), 5.86(1 \mathrm{H}, \mathrm{d}$, $J$ 4.2, C(5)H), 7.48-7.53 (2H, m, ArH), 7.65 (1H, dd, J 8.7, 1.7, $\operatorname{ArC}(2) H), 7.79-7.93$ (3H, m, $\mathrm{Ar} H), 8.15-8.23(1 \mathrm{H}, \mathrm{m}, \mathrm{Ar} H) ;{ }^{13} \mathrm{C}\left\{{ }^{1} \mathrm{H}\right\} \mathrm{NMR}\left(75 \mathrm{MHz}, \mathrm{CDCl}_{3}\right) \delta_{\mathrm{C}}: 14.3\left(\mathrm{CH}_{2} \mathrm{CH}_{3}\right), 15.0$ $\left(\mathrm{C}(3) \mathrm{CH}_{3}\right), 35.7(C(3) \mathrm{H}), 45.0(C(4) \mathrm{H}), 62.0\left(\mathrm{OCH}_{2}\right), 98.0(C(5) \mathrm{H}), 122.0(\mathrm{ArCH}), 124.5$ $(\mathrm{ArCH}), 126.8(\mathrm{ArCH}), 127.0(\mathrm{ArCH}), 127.7(\mathrm{ArCH}), 128.4(\mathrm{ArCH}), 128.8(\mathrm{ArCH}), 128.8$ $(\mathrm{ArC}), 133.1(\mathrm{ArC}), 133.7(\mathrm{ArC}), 150.9(C(6)), 170.3\left(C \mathrm{O}_{2} \mathrm{Et}\right), 171.5(C(2))$; HRMS $\left(\mathrm{NSI}^{+}\right)$ $\mathrm{C}_{19} \mathrm{H}_{19} \mathrm{O}_{4}[\mathrm{M}+\mathrm{H}]^{+}$found 311.1282, requires 311.1278 (+1.3 ppm).

\section{Acknowledgements}

We thank the European Research Council under the European Union's Seventh Framework Programme (FP7/2007-2013) ERC Grant Agreement No. 279850 (J.E.T. and A.T.D.) as well as the EPSRC and AstraZeneca (Case award to J.J.D.) for financial support. A.D.S. thanks the Royal Society for a Wolfson Merit Award. We also thank the EPSRC UK National Mass Spectrometry Facility at Swansea University.

\section{Supplementary data}

Supplementary data associated with this article can be found, in the online version, at $\mathrm{xxx}$. 


\section{References}

1. For reviews of NHC organocatalysis, see: (a) Enders, D.; Niemeier, O.; Henseler, A. Chem. Rev. 2007, 107, 5606-5655; (b) Marion, N.; Díez-González, S.; Nolan, S. P. Angew. Chem. Int. Ed. 2007, 46, 2988-3000; (c) Moore, J. L.; Rovis, T. Top. Curr. Chem. 2010, 291, 77-144; (d) Ryan, S. J.; Candish, L.; Lupton, D. W. Chem. Soc. Rev. 2013, 42, 4906-4917; (e) Flanigan, D. M.; Romanov-Michailidis, F.; White, N. A.; Rovis, T. Chem. Rev. 2015, 115, 9307-9387.

2. For seminal examples, see: (a) Zhang, Y.-R.; He, L.; Wu, X.; Shao, P.-L.; Ye, S. Org. Lett. 2008, 10, 277-280; (b) Duguet, N.; Campbell, C. D.; Slawin, A. M. Z.; Smith, A. D. Org. Biomol. Chem. 2008, 6, 1108-1113.

3. For seminal examples, see: (a) Zhao, X.; Ruhl, K. E.; Rovis, T. Angew. Chem. Int. Ed. 2012, 51, 12330-12333; (b) Mo, J.; Yang, R.; Chen, X.; Tiwari, B.; Chi, Y. R. Org. Lett. 2013, 15, 50-53; (c) Dong, X.; Yang, W.; Hu, W.; Sun, J. Angew. Chem. Int. Ed. 2015, 54, 660-663; (d) Lin, L.; Yang, Y.; Wang, M.; Lai, L.; Guo, Y.; Wang, R. Chem. Commun. 2015, 51, 8134-8137.

4. For seminal examples, see: (a) Hao, L.; Du, Y.; Lv, H.; Chen, X. K.; Jiang, H. S.; Shao, Y. L.; Chi, Y. R. Org. Lett. 2012, 14, 2154-2157; (b) Lee, A.; Younai, A.; Price, C. K.; Izquierdo, J.; Mishra, R. K.; Scheidt, K. A. J. Am. Chem. Soc. 2014, 136, 10589-10592; (c) Cheng, J.-T.; Chen, X.-Y.; Ye, S. Org. Biomol. Chem. 2015, 13, 1313-1316; (d) Lee, A.; Scheidt, K. A. Chem. Commun. 2015, 51, 3407-3410.

5. For reviews on NHC-catalysed redox reactions of $\alpha$-reducible aldehydes, see: (a) Rong, Z. Q.; Zhang, W.; Yang, G. Q.; You, S. L. Curr. Org. Chem. 2011, 15, 3077-3090; (b) Vora, H. U.; Wheeler, P.; Rovis, T. Adv. Synth. Catal. 2012, 354, 1617-1639; (c) Douglas, J.; Churchill, G.; Smith, A. D. Synthesis 2012, 44, 2295-2309.

6. (a) Reynolds, N. T.; Rovis, T. J. Am. Chem. Soc. 2005, 127, 16406-16407; (b) He, M.; Uc, G. J.; Bode, J. W. J. Am. Chem. Soc. 2006, 128, 15088-15089; (c) He, M.; Beahm, B. J.; Bode, J. W. Org. Lett. 2008, 10, 3817-3820; (d) Kobayashi, S.; Kinoshita, T.; Uehara, H.; Sudo, T.; Ryu, I. Org. Lett. 2009, 11, 3934-3937; (e) Yang, L. M.; Wang, F.; Chua, P. J.; Lv, Y. B.; Zhong, L. J.; Zhong, G. F. Org. Lett. 2012, 14, 2894-2897; (f) Ni, Q.; Zhang, H.; Grossmann, A.; Loh, C. C. J.; Merkens, C.; Enders, D. Angew. Chem. Int. Ed. 2013, 52, 13562-13566; (g) Dong, X.; Yang, W.; Hu, W.; Sun, J. Angew. Chem. Int. Ed. 2015, 54, 660-663; (h) Gao, Z.-H.; Chen, X.-Y.; Cheng, J.-T.; Liao, W.L.; Ye, S. Chem. Commun. 2015, 51, 9328-9331; (i) Ni, Q.; Song, X.; Xiong, J.; Raabe, 
G.; Enders, D. Chem. Commun. 2015, 51, 1263-1266; (j) Song, X.; Ni, Q.; Zhu, C.; Raabe, G.; Enders, D. Synthesis 2015, 47, 421-428.

7. For seminal examples, see: (a) He, M.; Struble, J. R.; Bode, J. W. J. Am. Chem. Soc. 2006, 128, 8418-8420; (b) Phillips, E. M.; Wadamoto, M.; Chan, A.; Scheidt, K. A. Angew. Chem. Int. Ed. 2007, 46, 3107-3110; (c) Wadamoto, M.; Phillips, E. M.; Reynolds, T. E.; Scheidt, K. A. J. Am. Chem. Soc. 2007, 129, 10098-10099; (d) Kaeobamrung, J.; Kozlowski, M. C.; Bode, J. W. Proc. Natl. Acad. Sci. U.S.A. 2010, 107, 20661-20665.

8. (a) Kawanaka, Y.; Phillips, E. M.; Scheidt, K. A. J. Am. Chem. Soc. 2009, 131, 1802818029; (b) Phillips, E. M.; Wadamoto, M.; Roth, H. S.; Ott, A. W.; Scheidt, K. A. Org. Lett. 2009, 11, 105-108.

9. (a) Li, G.-Q.; Dai, L.-X.; You, S.-L. Org. Lett. 2009, 11, 1623-1625; (b) Lv, H.; Mo, J. M.; Fang, X. Q.; Chi, Y. R. Org. Lett. 2011, 13, 5366-5369.

10. (a) Ling, K. B.; Smith, A. D. Chem. Commun. 2011, 47, 373-375; (b) Davies, A. T.; Taylor, J. E.; Douglas, J.; Collett, C. J.; Morrill, L. C.; Fallan, C.; Slawin, A. M. Z.; Churchill, G.; Smith, A. D. J. Org. Chem. 2013, 78, 9243-9257; (c) Taylor, J. E.; Daniels, D. S. B.; Smith, A. D. Org. Lett. 2013, 15, 6058-6061; (d) Davies, A. T.; Pickett, P. M.; Slawin, A. M. Z.; Smith, A. D. ACS Catal. 2014, 4, 2696-2700; (e) Attaba, N.; Taylor, J. E.; Slawin, A. M. Z.; Smith, A. D. J. Org. Chem. 2015, 80, 9728 9739; (f) Davies, A. T.; Slawin, A. M. Z.; Smith, A. D. Chem. Eur. J. 2015, 21, 1894418948; (g) Kerr, R. W. F.; Greenhalgh, M. D.; Slawin, A. M. Z.; Arnold, P. L.; Smith, A. D. Tetrahedron: Asymmetry 2016, DOI: 10.1016/j.tetasy.2016.10.012.

11. $\alpha$-Aroyloxyaldehydes are readily prepared on gram-scale from the parent aldehyde and 4-nitrobenzoic acid under oxidative conditions using a modified procedure of that described by Ishihara and co-workers, see: Uyanik, M.; Suzuki, D.; Yasui, T.; Ishihara, K. Angew. Chem. Int. Ed. 2011, 50, 5331-5334.

12. Authentic racemic samples of all dihydropyranones were prepared using $( \pm)-\mathbf{2}$.

13. Related isomerisation products were also observed in the NHC-catalysed reaction of disubstituted ketenes with $\beta, \gamma$-unsaturated $\alpha$-ketoesters, see: Leckie, S. M.; Brown, T. B.; Pryde, D.; Lebl, T.; Slawin, A. M. Z.; Smith, A. D. Org. Biomol. Chem. 2013, 11, 3230-3246.

14. The er of the products arising from epimerisation and isomerisation of syn-9 could not be determined as the complex mixtures were inseparable by HPLC analysis. 
15. (a) Allen, S. E.; Mahatthananchai, J.; Bode, J. W.; Kozlowski, M. C. J. Am. Chem. Soc. 2012, 134, 12098-12103; (b) Mahatthananchai, J.; Bode, J. W. Acc. Chem. Res. 2014, 47, 696-707.

16. Srivastava, B. K.; Joharapurkar, A.; Raval, S.; Patel, J. Z.; Soni, R.; Raval, P.; Gite, A.; Goswami, A.; Sadhwani, N.; Gandhi, N.; Patel, H.; Mishra, B.; Solanki, M.; Pandey, B.; Jain, M. R.; Patel, P. R. J. Med. Chem. 2007, 50, 5951-5966.

17. Meng, Q.; Zhu, L.; Zhang, Z. J. Org. Chem. 2008, 73, 7209-7212.

18. Belmessieri, D.; Morrill, L. C.; Simal, C.; Slawin, A. M. Z.; Smith, A. D. J. Am. Chem. Soc. 2011, 133, 2714-2720

19. Chappelle, M. R.; Harding, J. R.; Kent, B. B.; Jones, J. R.; Lu, S.-Y.; Morgan, A. D. J. Labelled Compd. Radiopharm. 2003, 46, 567-574.

20. Zhdanko, A.; Schmauder, A.; Ma, C. I.; Sibley, L. D.; Sept, D.; Sasse, F.; Maier, M. E. Chem. Eur. J. 2011, 17, 13349-13357.

21. Lu, H.-H.; Wang, X.-F.; Yao, C.-J.; Zhang, J.-M.; Wu, H.; Xiao, W.-J. Chem. Commun. 2009, 4251-4253.

22. Barluenga, J.; Lonzi, G.; Riesgo, L.; Tomás, M.; López, L. A. J. Am. Chem. Soc. 2011, 133, 18138-18141. 